\title{
Resolving the Interstellar Medium in the Nuclear Region of Two $z=5.78$ Quasar Host Galaxies with ALMA
}

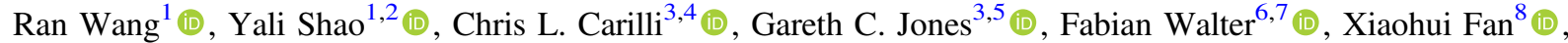 \\ Dominik A. Riechers ${ }^{9}$ (i), Roberto Decarli ${ }^{6,10}$ (i), Frank Bertoldi ${ }^{11}$ (i), Jeff Wagg ${ }^{12}$, Michael A. Strauss ${ }^{13}$ (iD), Alain Omont ${ }^{14}$ (iD), \\ Pierre $\operatorname{Cox}^{14}$, Linhua Jiang ${ }^{1}$ (1), Desika Narayanan ${ }^{15,16,17}$ (10), Karl M. Menten ${ }^{18}$ (1) , and Bram P. Venemans ${ }^{6}$ (1) \\ ${ }^{1}$ Kavli Institute of Astronomy and Astrophysics at Peking University, No. 5 Yiheyuan Road, Haidian District, Beijing, 100871, People's Republic of China \\ rwangkiaa@pku.edu.cn \\ ${ }^{2}$ Department of Astronomy, School of Physics, Peking University, Beijing 100871, People's Republic of China \\ ${ }^{3}$ Cavendish Laboratory, University of Cambridge, 19 J. J. Thomson Avenue, Cambridge CB3 0HE, UK \\ ${ }^{4}$ National Radio Astronomy Observatory, Socorro, NM 87801-0387, USA \\ ${ }^{5}$ Kavli Institute for Cosmology, University of Cambridge, Madingley Road, Cambridge CB3 0HA, UK \\ ${ }^{6}$ Max-Planck-Institut for Astronomie, Königstuhl 17, D-69117 Heidelberg, Germany \\ ${ }^{7}$ Astronomy Department, California Institute of Technology, MC105-24, Pasadena, CA 91125, USA \\ ${ }^{8}$ Steward Observatory, University of Arizona, 933 North Cherry Avenue, Tucson, AZ 85721, USA \\ 9 Department of Astronomy, Cornell University, 220 Space Sciences Building, Ithaca, NY 14853, USA \\ ${ }^{10}$ INAF-Osservatorio di Astrofisica e Scienza dello Spazio, via Gobetti 93/3, I-40129, Bologna, Italy \\ ${ }^{11}$ Argelander-Institut für Astronomie, University at Bonn, Auf dem Hügel 71, D-53121 Bonn, Germany \\ ${ }^{12}$ SKA Organization, Lower Withington, Macclesfield, Cheshire SK11 9DL, UK
Department of Astrophysical Sciences, Princeton University, Princeton, NJ 08544, USA \\ ${ }^{14}$ Institut d'Astrophysique de Paris, CNRS and Universite Pierre et Marie Curie, 98 b boulevard Arago, F-75014 Paris, France \\ ${ }^{15}$ Department of Astronomy, University of Florida, 211 Bryant Space Sciences Center, Gainesville, FL 32611, USA \\ ${ }^{16}$ University of Florida Informatics Institute, 432 Newell Drive, CISE Bldg E251, Gainesville, FL 32611, USA \\ ${ }^{17}$ Cosmic Dawn Center (DAWN), Niels Bohr Institute, University of Copenhagen, Juliane Maries vej 30, DK-2100 Copenhagen, Denmark \\ ${ }^{18}$ Max-Planck-Institut für Radioastronomie, Auf dem Hügel 69, D-53121 Bonn, Germany \\ Received 2018 August 17; revised 2019 October 6; accepted 2019 October 10; published 2019 December 9
}

\begin{abstract}
We present ALMA observations of the [C II] $158 \mu \mathrm{m}$ fine structure line and dust continuum emission from two quasars, SDSS J104433.04-012502.2 and SDSS J012958.51-003539.7, at $z=5.78$. The ALMA observations at 0 ." 2 resolution map the dust and gas on kiloparsec scales. The spatially resolved emission shows a similar trend of decreasing [C II]-far-infrared (FIR) ratios with increasing FIR surface brightnesses as was found in the infrared luminous galaxies with intense star formation. We confirm the velocity gradients of [C II] emission found previously in SDSS J0129-0035. No clear evidence of order motion is detected in SDSS J1044-0125. The velocity maps and position-velocity diagrams also suggest turbulent gas clumps in both objects. We tentatively detect a [C II] peak offset $4.9 \mathrm{kpc}$ to the east of SDSS J1044-0125. This may be associated with an infalling companion, or node of gas outflow. All these results suggest significant dynamical evolution of the interstellar medium in the nuclear region of these young quasar-starburst systems. We fit the velocity map of the [C II] emission from SDSS J0129-0035 with a rotating disk model. The result suggests a face-on system with an inclination angle of $16^{\circ} \pm 20^{\circ}$ and constrains the lower limit of the host galaxy dynamical mass to be $2.6 \times 10^{10} M_{\odot}$ within the [C II] emitting region. It is likely that SDSS J0129-0035, as well as other young quasars with supermassive black hole masses on the order of $10^{7} M_{\odot}$ to $10^{8} M_{\odot}$, falls close to the black hole and host galaxy mass relation defined by local galaxies.
\end{abstract}

Key words: galaxies: active - galaxies: evolution - galaxies: high-redshift

\section{Introduction}

Quasars at high redshifts provide the best opportunity to study the formation and early evolution of the first supermassive black holes (SMBHs) and their host galaxies. Due to the great success of wide-field optical and near-infrared surveys in the last two decades, more than two hundred quasars have been discovered at $z \geqslant 5.7$, an epoch close to the end of cosmic reionization (e.g., Fan et al. 2006; Jiang et al. 2009, 2016; Mortlock et al. 2011; Venemans et al. 2013; Bañados et al. 2016, 2018; Matsuoka et al. 2016, 2018b, 2018a; Chehade et al. 2018). This quasar sample covers a wide range of SMBH masses and quasar luminosities, including the most massive sources with SMBH mass above $10^{10} M_{\odot}$ (e.g., Fan et al. 2000; Wu et al. 2015) as well as objects with SMBH masses of a few $10^{7}-10^{8} M_{\odot}$ (Jiang et al. 2009; Willott et al. 2010; Matsuoka et al. 2016, 2018b, 2018a) which are more similar to the common quasar population observed at lower redshifts.
The dust and gas in the host galaxies of these earliest quasars have been studied at submillimeter and millimeter wavelengths using thermal continuum emission, molecular (mostly $\mathrm{CO}$ ), and fine structure lines, such as the [C II] $158 \mu \mathrm{m}$ emission line (Bertoldi et al. 2003a, 2003b; Petric et al. 2003; Priddey et al. 2003; Robson et al. 2004; Walter et al. 2004, 2009; Wang et al. 2008, 2010, 2013; Riechers et al. 2009; Omont et al. 2013; Bañados et al. 2015; Willott et al. 2015, 2017; Venemans et al. 2016, 2017a, 2017b, 2017c; Decarli et al. 2017, 2018; Izumi et al. 2018). Dust continuum surveys at $\sim 0.5-1 \mathrm{mJy} \mathrm{rms}$ sensitivity have detected the most far-infrared (FIR)-luminous objects, with FIR luminosities of a few $\times 10^{12}$ to $10^{13} L_{\odot}$ and dust masses of $>10^{8} M_{\odot}$ (Bertoldi et al. 2003a; Priddey et al. 2003; Robson et al. 2004; Wang et al. 2007, 2008). These FIR luminous quasars are bright in $\mathrm{CO}$ line emission and [C II] $158 \mu \mathrm{m}$ fine structure line emission, indicating molecular gas with masses of the order of $10^{10} M_{\odot}$ and bursts of star 
formation in the nuclear region, coeval with the SMBH accretion and quasar activity (Bertoldi et al. 2003b; Maiolino et al. 2005; Carilli et al. 2007; Wang et al. 2013; Venemans et al. 2016; Decarli et al. 2017, 2018).

In recent years, the Atacama Large Millimeter/submillimeter Array (ALMA) has carried out comprehensive surveys of the [C II] $158 \mu \mathrm{m}$ fine structure line in high- $z$ quasars. For example, Decarli et al. (2018) detected [C II] in 85\% of 27 optically selected quasars at $z>5.94$. This line is an important coolant that traces the ionized and neutral interstellar medium (ISM) and star-forming activities (Herrera-Camus et al. 2015). The detection of [C II] line and dust continuum emission has revealed a wide range of star formation rates, from a few $10 M_{\odot} \mathrm{yr}^{-1}$ to $\geqslant 1000 M_{\odot} \mathrm{yr}^{-1}$ (Decarli et al. 2018; Izumi et al. 2018). The [C II]-FIR luminosity ratios of these quasar hosts range from $10^{-4}$ to a few $\times 10^{-3}$ (Maiolino et al. 2005; Wang et al. 2013; Willott et al. 2017; Decarli et al. 2018), following the trend of decreasing [C II]-FIR ratio with increasing FIR luminosities found with the IR luminous star-forming systems (Malhotra et al. 2001; Luhman et al. 2003; Hailey-Dunsheath et al. 2010; Stacey et al. 2010; Graciá-Carpio et al. 2011; DíazSantos et al. 2013, 2017; Muñoz \& Oh 2016; Smith et al. 2017; Decarli et al. 2018; Gullberg et al. 2018; Rybak et al. 2019). In particular, the quasar hosts with high FIR luminosities of a few $10^{12}$ to $10^{13} L_{\odot}$ show low [C II]-FIR ratios similar to that found in the ultraluminous infrared galaxies (ULIRGs) and submillimeter galaxies (SMGs; Luhman et al. 2003; Díaz-Santos et al. 2013, 2017; Wang et al. 2013; Rybak et al. 2019). The [C II]-FIR ratio is suggested to be related to the local conditions of the ISM; e.g., a decrease of [C II]-FIR ratio could be due to a high gas temperature (i.e., $T>\Delta E / k \sim 91 \mathrm{~K}$, $\Delta E$ is the energy separation of the two levels of the [C II] $158 \mu \mathrm{m}$ transition) where the upper level of the [C II] transition is saturated (Muñoz \& Oh 2016), or a high gas surface density (e.g., the typical density in compact ULIRGs and SMGs) where more gas is in the molecular phase (Narayanan \& Krumholz 2017). It is interesting to see how the resolved distributions of the line and continuum surface brightnesses and emission ratios in these FIR luminous quasar hosts compare to that in the ULIRGs and SMGs (Smith et al. 2017; Gullberg et al. 2018; Rybak et al. 2019).

The [C II] $158 \mu \mathrm{m}$ and molecular CO lines are bright tracers of the gas content that could be detected from distant galaxies (Solomon \& Vanden Bout 2005; Carilli \& Walter 2013). Measurements of the line widths, profiles, and velocity maps of these emission lines reveal important information on the gas kinematics (Walter et al. 2004; Ivison et al. 2011; Maiolino et al. 2012; Wang et al. 2013; Venemans et al. 2016, 2019). Observations of the $[\mathrm{C} \mathrm{II}]$ and $\mathrm{CO}$ emission lines from the young quasar hosts at $z \geqslant 5.7$ at subarcsecond to arcsecond resolution show a range of kinematic properties, including velocity gradients of ordered motion (Willott et al. 2013, 2017; Venemans et al. 2016; Shao et al. 2017; Feruglio et al. 2018), large velocity dispersion/turbulence (e.g., SDSS J231038.88 +185519.7 , Feruglio et al. 2018), gas outflows (e.g., SDSS J114816.64+515250.3, Maiolino et al. 2012; Cicone et al. 2015), and very compact sources with no evidence of rotation (e.g., ULAS J112001.48+064124.3, Venemans et al. 2017c). All these submillimeter/millimeter (submm/mm) observations argue for an early phase of SMBH-galaxy coevolution in the $z \geqslant 5.7$ quasar sample. Deep imaging of the gas component at the kiloparsec scale is required to fully resolve the gas distribution and kinematic properties in these systems.

In this paper, we present the observations, results, and conclusion for two out of three quasars from our ALMA Cycle 1 program. Our team has carried out ALMA observations at 0!" 2-0." 3 angular resolution to image the [C II] line emission from the host galaxies of three quasars, ULAS J131911.29+095051.4 (hereafter ULAS J1319+0950), SDSS J104433.04-012502.2 (hereafter SDSS J1044-0125), and SDSS J012958.51-003539.7 (hereafter SDSS J0129-0035) at $z \geqslant 5$.7. These three objects are among the brightest dust continuum, and [C II] detections at $z \sim 6$ (Wang et al. 2013), with FIR luminosities of $5 \sim 10 \times 10^{12} L_{\odot}$ and $^{19}$ [C II] luminosities of $1.5 \sim 4.4 \times 10^{9} L_{\odot}$ (Wang et al. 2013), suggesting massive star formation in the quasar hosts. The three objects are different in quasar UV luminosities and SMBH masses. SDSS J1044-0125 and ULAS J1319+0950 are among the most optically luminous quasars at $z \sim 6$ with rest-frame $1450 \AA$ apparent magnitudes of $m_{1450}=19.21$ and 19.65, respectively (Fan et al. 2000; Mortlock et al. 2009). The SMBH mass is $(2.7 \pm 0.6) \times 10^{9} M_{\odot}$ for ULAS J1319+0950 (Shao et al. 2017) and $(5.6 \pm 0.6) \times 10^{9} M_{\odot}$ for SDSS J1044 -0125 (Shen et al. 2019). SDSS J0129-0035 is much fainter in quasar emission with $m_{1450}=22.28$ (Jiang et al. 2009). If Eddington accretion is assumed (Willott et al. 2010), the SMBH mass is $1.7 \times 10^{8} M_{\odot}$. These objects provide an ideal sample for a pilot study of the ISM distribution and dynamics in the earliest SMBH-starburst systems over a range of quasar luminosities.

We report our ALMA [C II] line imaging of ULAS J1319 +0950 at 0.! 3 resolution in Shao et al. (2017). The [C II] velocity map of this object shows clear velocity gradients and the spectrum appears to be broad (FWHM $\sim 540 \mathrm{~km} \mathrm{~s}^{-1}$ ) and flat at the top, suggesting that the $[\mathrm{C} \mathrm{II}]$-emitting gas is mainly distributed in an inclined rotating disk extending to a radius of $\sim 3.2 \mathrm{kpc}$. We performed dynamical modeling of the gas velocity field and measured the disk inclination angle and rotation velocity of the atomic/ionized gas in the nuclear region. The derived host galaxy dynamical mass is $1.3 \times 10^{11} M_{\odot}$, suggesting a black hole-to-host galaxy mass ratio of $\sim 0.02$, i.e., about four times higher than the value expected from the black hole-bulge mass relation of local galaxies (Kormendy \& Ho 2013).

We here present ALMA [C II] imaging of the remaining two quasars, SDSS J0129-0035 and SDSS J1044-0125. We describe the observations in Section 2, present the results in Section 3, discuss the surface brightnesses of the [C II] and dust emission and gas kinematic properties in Section 4, and summarize the main conclusions in Section 5. We adopt a $\Lambda \mathrm{CDM}$ cosmology with $H_{0}=71 \mathrm{~km} \mathrm{~s}^{-1} \mathrm{Mpc}^{-1}, \Omega_{\mathrm{M}}=0.27$, and $\Omega_{\Lambda}=0.73$ throughout this paper (Spergel et al. 2007).

\section{Observations}

We carried out observations of the [C II] $158 \mu \mathrm{m}$ emission line from SDSS J0129-0035 and SDSS J1044-0125 using the Band 7 receiver on ALMA in Cycle 1 (Program ID: 2012.1.00240.S). The data was taken in 2015 July and August in the C34-6 configuration with $3212 \mathrm{~m}$ diameter antennas and a maximum baseline of $1.6 \mathrm{~km}$. This results in a full width at half maximum (FWHM) synthesized beam size of $\sim 0$ ". 2 using

\footnotetext{
${ }^{19} \mathrm{We}$ adopt the rest-frame wavelength range of $42.5-122.5 \mu \mathrm{m}$ for FIR luminosity calculation
} 

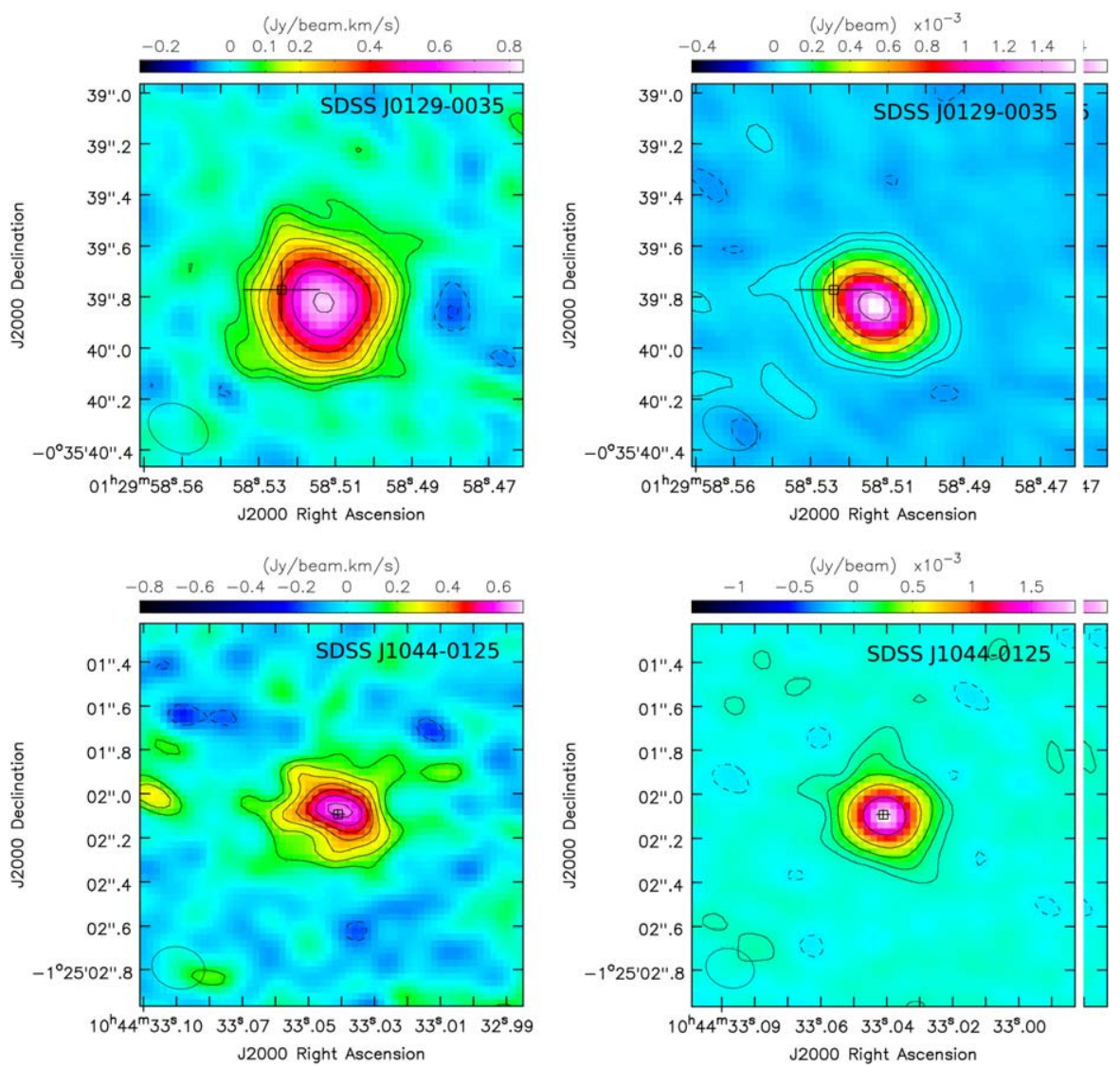

Figure 1. The upper panels show the [C II] line velocity-integrated map (left) and continuum intensity map (right) of SDSS J0129-0035. The contours are [ $-2 \sqrt{2}$, $-2,2,2 \sqrt{2}, 4,4 \sqrt{2}, 8,8 \sqrt{2}, 16,16 \sqrt{2}] \times 0.034 \mathrm{Jy}_{\text {beam }}{ }^{-1} \mathrm{~km} \mathrm{~s}^{-1}$ for the line, and $[-2,2,4,8,16,32,64] \times 0.021 \mathrm{mJy}^{-1}$ beam ${ }^{-1}$ for the continuum. The ellipse on the bottom left denotes the FWHM synthesized beam size, which is 0 !" $25 \times 0$ !" 18 for the line and 0 ! $22 \times 0$ ! 16 for the continuum. The black square with a cross represents the position of the optical quasar, and the cross bars represent the uncertainties of 149 mas in R.A. and 114 mas in decl. measured from Gaia. The lower panels show the $[\mathrm{C}$ II] line velocity-integrated intensity map (left) and continuum map (right) of SDSS J1044-0125. The contours are [-2, 2, 3, 4, 5, 6, 7, $8] \times 0.076 \mathrm{Jy}_{\text {beam }}^{-1} \mathrm{~km} \mathrm{~s}^{-1}$ for the line, and $[-2,2,4,8,16,32] \times 0.04 \mathrm{mJy}$ beam ${ }^{-1}$ for the continuum. The FWHM beam sizes shown on the bottom left are 0 ". $24 \times 0$ "! 19 for the line and 0 ". $22 \times 0$ ". 18 for the continuum. The black square with a cross denotes the optical quasar position and the cross bars represent the uncertainties of 27 mas in R.A. and 20 mas in decl. from Gaia.

robust weighting, which corresponds to $1.2 \mathrm{kpc}$ at $z \sim 5.8$. We tuned the four $1.785 \mathrm{GHz}$ spectral windows with one window centered on the redshifted [C II] line frequencies of the two targets and the other three windows observing the continuum. The correlator channel width is $15.625 \mathrm{MHz}$, corresponding to a velocity resolution of $\sim 17 \mathrm{~km} \mathrm{~s}^{-1}$. We checked the phase every $5 \sim 7$ minutes on nearby calibrators, and the flux scale was calibrated on Ceres and $\mathrm{J} 1058+015$ with calibration uncertainties less than $10 \%$. The total on-source time is about 80 minutes for SDSS J0129-0035 and 75 minutes for SDSS J1044-0125.

The data were calibrated using the pipeline of the Common Astronomy Software Application (CASA) Version 4.3.1. We then combined the data with that obtained from previous ALMA Cycle 0 observations (Program ID: 2011.0.00206.S, Wang et al. 2013). The ALMA Cycle 0 data alone has a lower angular resolution of $0 . " 6-0 . ! 7$, and $1 \sigma \mathrm{rms}$ sensitivities of $0.35 \mathrm{mJy}$ beam $^{-1}$ for SDSS J0129-0035 and $0.65 \mathrm{mJy}^{-1}$ for SDSS J1044-0125 in each $67 \mathrm{~km} \mathrm{~s}^{-1}$ channel (Wang et al. 2013). By including the Cycle 0 data, we could better sample the $u v$ plane at different scale and improve the sensitivity at short $u v$-distance. As the Cycle 0 data were calibrated using a much earlier version of CASA, we corrected the weights before combining with the Cycle 1 data. ${ }^{20}$ We checked the amplitudes of visibility data from the two observations at overlapping $u v$ distance, and the flux scales are consistent with each other within the calibration uncertainties. We averaged, in the visibility domain, the emission in line-free channels to form a "pseudo" continuum data set which we then subtracted from the $u v$-data to obtain a line-only data set. We made the continuum image with the data from three line-free spectral windows and made the line image cubes with the continuumsubtracted visibility data set. The images were cleaned using the CLEAN task in CASA using robust $=0.5$ for weighting. The typical rms noise is $0.20 \mathrm{mJy}^{\mathrm{beam}}{ }^{-1}$ per $17 \mathrm{~km} \mathrm{~s}^{-1}$ channel for the final image cube of SDSS J0129-0035, and $0.36 \mathrm{mJy}_{\text {beam }}{ }^{-1}$ per $17 \mathrm{~km} \mathrm{~s}^{-1}$ channel for SDSS J1044-0125. The rms noise of the continuum is $21 \mu \mathrm{Jy}$ beam $^{-1}$ for SDSS J0129-0035, and $40 \mu \mathrm{Jy}_{\text {beam }}{ }^{-1}$ for SDSS J1044-0125.

\section{Results}

For each of the two objects, we integrated the [C II] emission over the line-emitting channels to obtain velocity-integrated maps and averaged the line-free channels to obtain the continuum maps

\footnotetext{
${ }^{20}$ https://casaguides.nrao.edu/index.php/DataWeightsAndCombination
} 

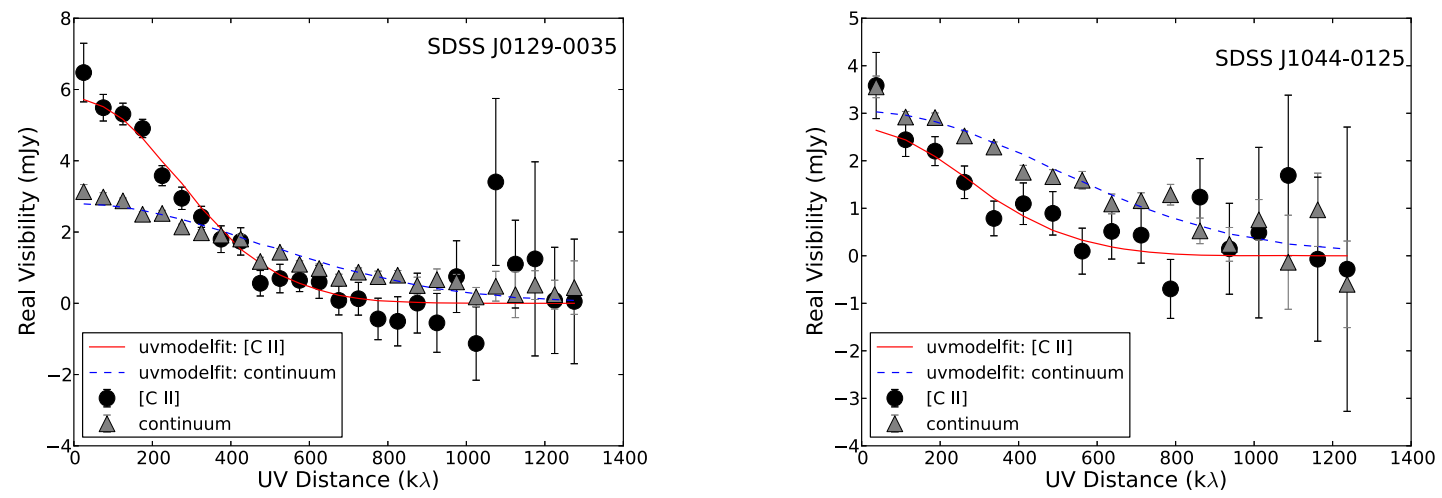

Figure 2. Visibility vs. $u v$ distance of the [C II] line (black squares) and continuum emission (gray triangles). We reset the phase center to the peak of the [C II] line emission (Table 1). The visibility data are radially binned with a bin size of $50 \mathrm{k} \lambda$ for SDSS J0129-0035 and $75 \mathrm{k} \lambda$ for SDSS J1044-0125. The error bars show the standard deviation of the mean value in each bin. We fit an elliptical Gaussian to the $u v$ data and the $u v$ models are shown as red solid and blue dashed lines for the line and continuum, respectively.

Table 1

Line and Continuum Parameters

\begin{tabular}{|c|c|c|}
\hline & SDSS J0129-0035 & SDSS J1044-0125 \\
\hline [C II] line flux $\left(\mathrm{Jy} \mathrm{km} \mathrm{s}^{-1}\right)$ & $2.11 \pm 0.09$ & $1.52 \pm 0.15$ \\
\hline Peak $\left(\mathrm{Jy} \mathrm{beam}^{-1} \mathrm{~km} \mathrm{~s}^{-1}\right)$ & $0.80 \pm 0.03$ & $0.64 \pm 0.08$ \\
\hline $\mathrm{FWHM}_{[\mathrm{C} \mathrm{II]}}\left(\mathrm{km} \mathrm{s}^{-1}\right)$ & $200 \pm 8$ & $440 \pm 40$ \\
\hline [C II] central position (J2000) & $01^{\mathrm{h}} 29^{\mathrm{m}} 58^{\mathrm{s}} .513-00^{\circ} 35^{\prime} 39^{\prime \prime} .83$ & $10^{\mathrm{h}} 44^{\mathrm{m}} 33^{\mathrm{s}} .042-01^{\circ} 25^{\prime} 02^{\prime \prime} 08$ \\
\hline Deconvolved FWHM [C II] size (imfit) & 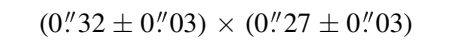 & $(0 " .41 \pm 0.07) \times(0 " 30 \pm 0$ ".06) \\
\hline FWHM [C II] size (uvmodelfit) & $\left(0 . " 32 \pm 0{ }^{\prime \prime} 01\right) \times\left(0 " \prime 26 \pm 0{ }^{\prime \prime} 01\right)$ & $\left(0 . " 32 \pm 0{ }^{\prime \prime} 02\right) \times\left(0 "\right.$ " $\left.26 \pm 0{ }^{\prime \prime} 03\right)$ \\
\hline$L_{[\mathrm{C} \text { I] }}\left(L_{\odot}\right)$ & $(1.96 \pm 0.08) \times 10^{9}$ & $(1.41 \pm 0.14) \times 10^{9}$ \\
\hline Continuum (mJy) & $2.82 \pm 0.04$ & $3.02 \pm 0.11$ \\
\hline Continuum central position (J2000) & $01^{\mathrm{h}} 29^{\mathrm{m}} 58^{\mathrm{s}} .513-00^{\mathrm{d}} 35^{\prime} 39^{\prime \prime} .84$ & $10^{\mathrm{h}} 44^{\mathrm{m}} 33^{\mathrm{s}} \cdot 040-01^{\mathrm{d}} 25^{\prime} 02^{\prime \prime} 10$ \\
\hline Deconvolved FWHM continuum size (imfit) & $\left(0 ! \prime 18 \pm 0 !^{\prime \prime} 01\right) \times\left(0 !^{\prime \prime} 16 \pm 0{ }^{\prime \prime} 01\right)$ & 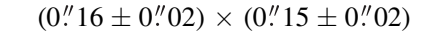 \\
\hline FWHM continuum size (uvmodelfit) & 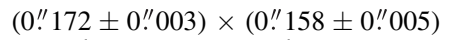 & $(0 ! 159 \pm 0$ " 005$) \times(0 " \prime 154 \pm 0$ ". 008$)$ \\
\hline Optical quasar position (J2000) & $01^{\mathrm{h}} 29^{\mathrm{m}} 58^{\mathrm{s}} .524-00^{\mathrm{d}} 35^{\prime} 39^{\prime \prime} 77$ & $10^{\mathrm{h}} 44^{\mathrm{m}} 33^{\mathrm{s}} \cdot 041-01^{\mathrm{d}} 25^{\prime} 02^{\prime \prime} 09$ \\
\hline Cycle 0 [C II] line flux ${ }^{\mathrm{a}}\left(\mathrm{Jy} \mathrm{km} \mathrm{s}^{-1}\right)$ & $1.99 \pm 0.12$ & $1.70 \pm 0.30$ \\
\hline Cycle 0 Continuum (mJy) & $2.57 \pm 0.06$ & $3.12 \pm 0.09$ \\
\hline
\end{tabular}

Note.

${ }^{a}$ Measurements of [C II] line flux and continuum flux density using the ALMA Cycle 0 data at 0. " 6 resolution from Wang et al. (2013). The calibration uncertainties are not included here, which is better than $15 \%-20 \%$ for the Cycle 0 data and better than $10 \%$ for the new data presented here.

(Figure 1). We then integrated the emission within the $2 \sigma$ contour region to get total [C II] line fluxes and continuum flux densities (Table 1). For SDSS J0129-0035, the [C II] velocity-integrated map has a peak of $0.80 \pm 0.03 \mathrm{Jy}_{\text {beam }}^{-1} \mathrm{~km} \mathrm{~s}^{-1}$ (signal-to-noise ratio, $\mathrm{S} / \mathrm{N}=23.5)$, which is about $38 \%$ of the total line flux. The continuum intensity map has a peak of $1.58 \pm 0.02 \mathrm{mJy}^{\text {beam }^{-1}}$ $(\mathrm{S} / \mathrm{N}=80), 56 \%$ of the integrated continuum flux density. For SDSS J1044-025, the [C II] peak is $0.64 \pm 0.08 \mathrm{Jy} \mathrm{beam}^{-1}$ $\mathrm{km} \mathrm{s}^{-1}$ (i.e., $\mathrm{S} / \mathrm{N}=8.4$ ). This is about $45 \%$ of the total line flux. The peak of the continuum emission is $1.88 \pm 0.04 \mathrm{mJy}_{\text {beam }}{ }^{-1}$ $(\mathrm{S} / \mathrm{N}=47.5)$, which is about $63 \%$ of the total continuum flux density. Thus, we consider the [C II] and continuum emission from both objects are resolved.

The visibilities versus $u v$-distance for the [C II] line and continuum are plotted in Figure 2. The data are radially averaged with a bin size of $50 \mathrm{k} \lambda$ for SDSS J0129-0035 and $75 \mathrm{k} \lambda$ for SDSS J1044-0125. The decrease of the visibility amplitudes toward longer $u v$-distance indicates that the line and continuum sources are resolved in the $u v$-plane. We performed elliptical Gaussian fitting to measure the sizes of the [C II] and continuum emission in both the image plane and the $u v$ plane, using imfit and uvmodelfit tasks in CASA, respectively. The results, together with the integrated line fluxes and continuum flux densities, are summarized in Table 1. The $u v$-models are also plotted in Figure 2. The Gaussian FWHM source sizes (deconvolved from the synthesized beam) and peak positions in the image plane are consistent with that from the $u v$-models within the fitting uncertainties. We adopt the fitting positions and deconvolved source sizes from imfit for the discussions in the rest of this paper.

The fitting uncertainties of the [C II] peak positions are $\sim 7$ mas in both R.A. and decl. for SDSS J0129-0035, and 25 mas for SDSS J1044-0125. The positional uncertainties for the continuum images are $<3$ mas for both objects. Thus, the peak positions of the [C II] line and continuum emission are consistent with each other within the fitting errors. We checked the coordinates of the optical quasars using the astrometric data from the Gaia mission (L. Lindegren et al. 2018, in preparation). For SDSS J0129 -0035 , the quasar coordinates are R.A. $=01^{\mathrm{h}} 29^{\mathrm{m}} 58.524$, and decl. $=-00^{\mathrm{d}} 35^{\prime} 39^{\prime \prime} .77$ with an uncertainty of 149 mas in R.A. and 114 mas in decl. Considering the coordinates' uncertainties, the positions of [C II] and dust continuum peaks are marginally consistent with that of the optical quasar (Figure 1). The quasar coordinates for SDSS $1044-0125$ are R.A. $=10^{\mathrm{h}} 44^{\mathrm{m}} 33^{\mathrm{s}} .041$, and decl. $=-01^{\mathrm{d}} 25^{\prime} 02^{\prime \prime} 09$, with an uncertainty of 27 mas in R.A. and 

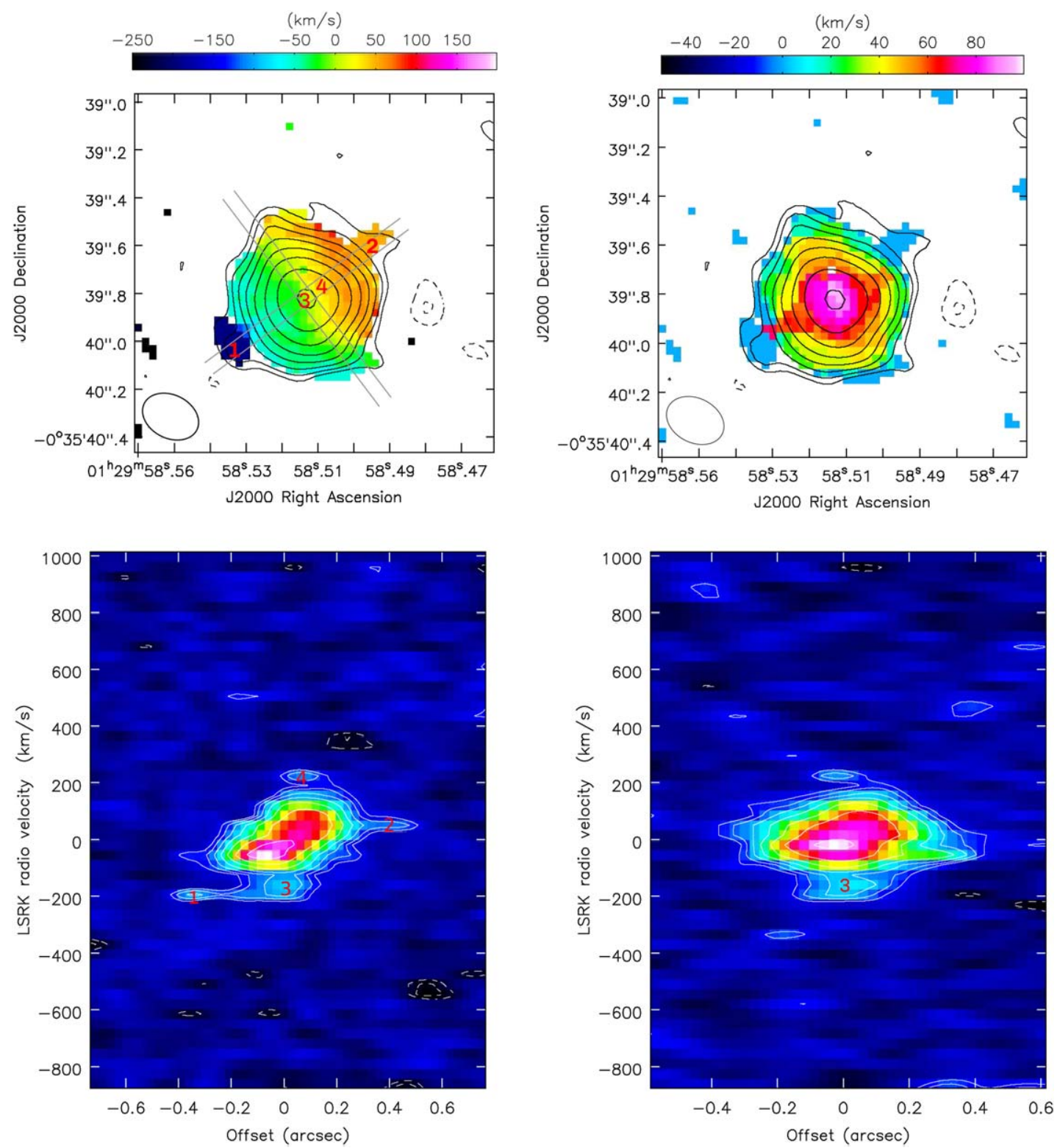

Figure 3. The upper panels show the intensity-weighted velocity and velocity dispersion maps of SDSS J0129-0035 (color maps). The maps are derived with the channel images including pixels at $\geqslant 3.5 \sigma$. The black contours are the $[\mathrm{C} \mathrm{II]} \mathrm{line} \mathrm{intensity} \mathrm{map} \mathrm{(same} \mathrm{as} \mathrm{Figure} \mathrm{1).} \mathrm{The} \mathrm{numbers} 1$ and 2 mark two substructures on the velocity map (see details in Section 3 and Section 4.2). The lower panels show the position-velocity (PV) diagrams along the direction of the two substructures $\left(\mathrm{PA}=127^{\circ}\right.$, left $)$ and the perpendicular direction $\left(\mathrm{PA}=37^{\circ}\right.$, right). The contour levels are $[-2,2,2 \sqrt{2}, 4,4 \sqrt{2}, 8,8 \sqrt{2}, 16] \times 0.2 \mathrm{mJy}$ beam ${ }^{-1}$. The zero velocity corresponds to the [C II] redshift of $z=5.7787$ (Wang et al. 2013). The central position for the PV diagrams is R.A. $01^{\mathrm{h}} 29^{\mathrm{m}} 58^{\mathrm{S}} .513$ decl. $-00^{\mathrm{d}} 35^{\prime} 39^{\prime \prime} \cdot 82$, and the slit width is 0. . 09 . There are also components in the PV diagrams that show velocities of $\pm 200 \mathrm{~km} \mathrm{~s}^{-1}$ within the central $<0$.! 2 region (see details in Section 3 and Section 4.2). We mark them as Nos. 3 and 4 in the PV diagrams. We also mark their position in the velocity map in the top left panel. The velocity map and PV diagrams suggest rotating motion of [C II]-emitting gas. The substructures (No. 1-4) may suggest additional clumps with complex kinematic properties.

20 mas in decl. This is consistent with the positions of the [C II] and dust continuum peaks of this object (Figure 1).

We finally check the results by comparing the measurements to those from the low-resolution ALMA Cycle 0 data (Table 1, Wang et al. 2013). The [C II] line fluxes and continuum flux densities integrated over the source regions of both objects are consistent within the calibration uncertainties with the previous values. The ALMA data at 0 "! 2 resolution reveal more detail on the spatial distribution and velocity field of the [C III-emitting gas component in the quasar host galaxies.

SDSS J0129-0035. We present the intensity-weighted velocity map and velocity dispersion map of the [C II] line emission in Figure 3. We adopt the [C II] redshift of
$\mathrm{z}_{[\mathrm{C} \mathrm{II}]}=5.7787$ as the zero velocity (Wang et al. 2013). The line is single-peaked and the velocity map shows a velocity gradient from the southeast to northwest direction. The velocity images also show two features at the edge of the [C II]-emitting region (marked as No. 1 and 2 in the velocity map, Figure 3). One has an intensity-weighted velocity of $-190 \mathrm{~km} \mathrm{~s}^{-1}$ at the southeast edge of the line-emitting region and the other has an intensity-weighted velocity of about $+50 \mathrm{~km} \mathrm{~s}^{-1}$ on the northwest part. There is no strong $(\geqslant 2 \sigma)$ dust emission detected in the regions of these two features. The intensity-weighted velocity dispersion map of the [C II] emission (upper right panel of Figure 3) also suggests a high velocity dispersion structure along the southeast-to-northwest direction, which 


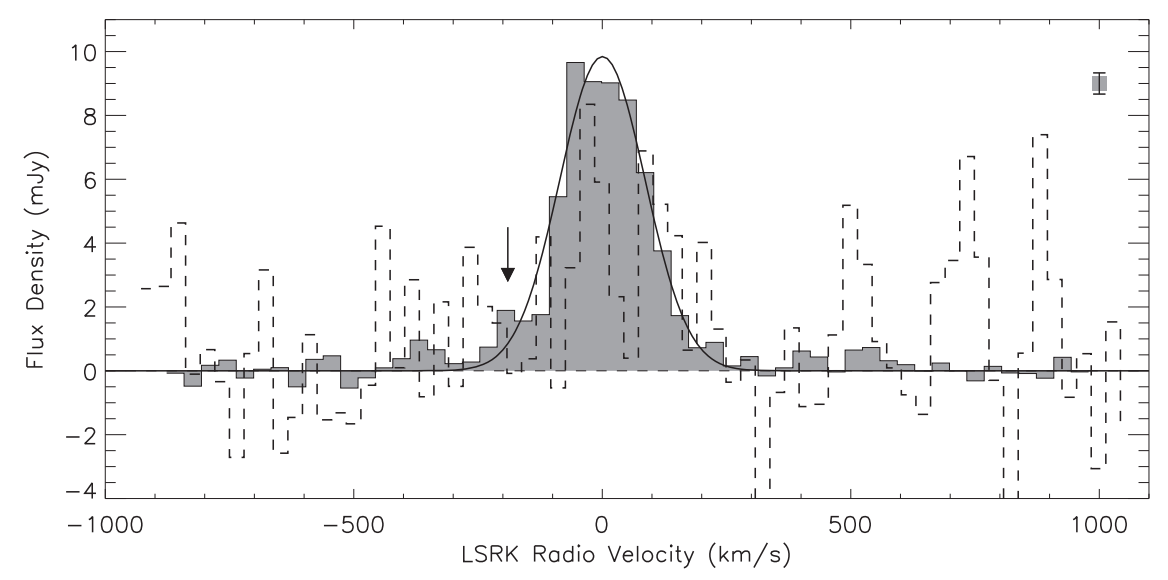

Figure 4. Gray histogram represents the [C II] line spectrum of SDSS J0129-0035 integrated over the line-emitting region within the $2 \sigma$ contour in the upper left panel of Figure 1. The error bar on the top right denotes the typical $1 \sigma \mathrm{rms}$ uncertainty per channel. The solid line shows a Gaussian line profile fitted to the [C II] line spectrum. The dashed line is the spectrum of the CO (6-5) line for this object scaled by a factor of 4 (Wang et al. 2011). The zero velocity corresponds to the [C II] redshift of $z=5.7787$ (Wang et al. 2013). The arrow marks a tentative excess on the blue wing of the [C II] line spectrum compared to the single-Gaussian line profile (see discussion in Section 3).

seems to extend from the center to the two features. A structure with such a velocity dispersion cannot be explained with beam smearing of a rotating disk.

The PV diagrams along the direction of the two features and the perpendicular direction are plotted in the lower panel of Figure 3. The major gas component in the PV diagrams is consistent with rotation. The two features mentioned above are presented at the regions around $\left(-0\right.$ ". $4,-190 \mathrm{~km} \mathrm{~s}^{-1}$, No. 1 in Figure 3) and (+0." $4,+50 \mathrm{~km} \mathrm{~s}^{-1}$, No. 2) in the bottom left panel. It is unclear if these two components are connected to the outer part of the rotating gas disk or not. The PV diagrams also show tentative peaks $(3 \sim 4 \sigma)$ with velocities of $\sim \pm 200 \mathrm{~km} \mathrm{~s}^{-1}$ within the central 0".2 (Nos. 3 and 4 in Figure 3). These components locate close to the center/peak of the $[\mathrm{C} \mathrm{II}]$-emitting region. However, the large velocities of $\sim \pm 200 \mathrm{~km} \mathrm{~s}^{-1}$ do not seem to follow the velocity field of the rotating component. These tentative peaks should be checked with deeper observations at better resolution (e.g., $\lesssim 0$ " 1 ), to confirm if they are real features and related to gas inflows/ outflows.

We plot the [C II] line spectrum integrated over the lineemitting region in Figure 4, compared to the CO (6-5) line spectrum published in Wang et al. (2011). Though the CO (6-5) line profile is poorly constrained due to the low $\mathrm{S} / \mathrm{N}$, the line widths and redshifts measured with the $[\mathrm{C} \mathrm{II}]$ and $\mathrm{CO}$ lines are consistent with each other within the uncertainties (Wang et al. 2011, 2013). Moreover, comparing to the Gaussian line profile that fit to the line spectrum, there is a tentative excess on the blue wing around $-200 \mathrm{~km} \mathrm{~s}^{-1}$ (arrow in Figure 4). This is likely to be related to the components seen in the velocity map and PV diagrams (e.g., Nos. 1 and 3).

SDSS J1044-0125. We calculate the intensity-weighted velocity and velocity dispersion maps with the $>4 \sigma$ pixels in each [C II] channel (Figure 5), which reveals the gas kinematics in the central high surface brightness part of the line-emitting region. The gas does show some velocity structure over this area. For example, the gas component to the northwest of the quasar position shows more positive velocities. However, the velocity map seems more complicated than what one would expect from a simple rotating disk. The PV diagrams also suggest a very turbulent gas velocity field; the gas is distributed over a compact region of about $2.4 \mathrm{kpc}$ (i.e., \pm 0 ". 2 ) with velocities from -200 to $\gtrsim 200 \mathrm{~km} \mathrm{~s}^{-1}$.

The integrated [C II] line spectrum shows multiple peaks and is not well fit by a single-Gaussian profile (Figure 6). We try to fit the line profile with three Gaussian components (A, B, and C). The fitting results of the line centers, FWHM widths, and fluxes are listed in Table 2. Here, we adopt the [C II] redshift of $z_{[\mathrm{C} \mathrm{II}]}=5.7847$ from the single-Gaussian fitting as the zero velocity (Wang et al. 2013). Wang et al. (2013) found a velocity offset between the Gaussian-fit line centers of the [C II] line and the CO (6-5) line for SDSS J1044-0125. With the new ALMA [C II] data in this work, we still see this offset. i.e., The CO (6-5) line overlaps only the blue part $\left(-300-0 \mathrm{~km} \mathrm{~s}^{-1}\right)$ of the [C II] line emission with a line center at around $-100 \pm 44 \mathrm{~km} \mathrm{~s}^{-1}$ (Wang et al. 2013) which is between the central velocities of components $\mathrm{A}$ and $\mathrm{B}$. The mismatch between the $[\mathrm{C} \mathrm{II}]$ and $\mathrm{CO}(6-5)$ spectra argues for complex gas components and kinematic properties in the nuclear region of SDSS J1044-0125 (see more discussion in Section 4.2).

On the velocity-integrated map of the [C II] line (lower left of Figure 1 and left of Figure 7), there is a tentative peak $0 . " 82$ (i.e., $4.9 \mathrm{kpc}$ ) to the east of the major component. The spectrum of the emission from this secondary peak position shows a positive signal from about -740 to $400 \mathrm{~km} \mathrm{~s}^{-1}$. We integrate the emission in this velocity range and show the intensity map in the left panel of Figure 7. The secondary peak is detected at about $5 \sigma$ on the image, with a flux of $0.52 \pm 0.10 \mathrm{Jy} \mathrm{km} \mathrm{s}^{-1}$. We also check the Cycle 0 data and the image at 0 ." 6 resolution does show an extension toward the secondary peak location (see red contours in the upper left panel of Figure 7). A further check shows that this component is also present in a continuum-unsubtracted image in the same velocity range, as well as the image using only Cycle 1 data. No emission is detected at this location with the data from the other three continuum spectral windows. Fitting this component with a Gaussian profile yields an FWHM line width of $750 \pm$ $190 \mathrm{~km} \mathrm{~s}^{-1}$, centered at $-190 \pm 80 \mathrm{~km} \mathrm{~s}^{-1}$. The [C II] luminosity derived with the peak intensity of this component is $(4.8 \pm 0.9) \times$ $10^{8} L_{\odot}$. 

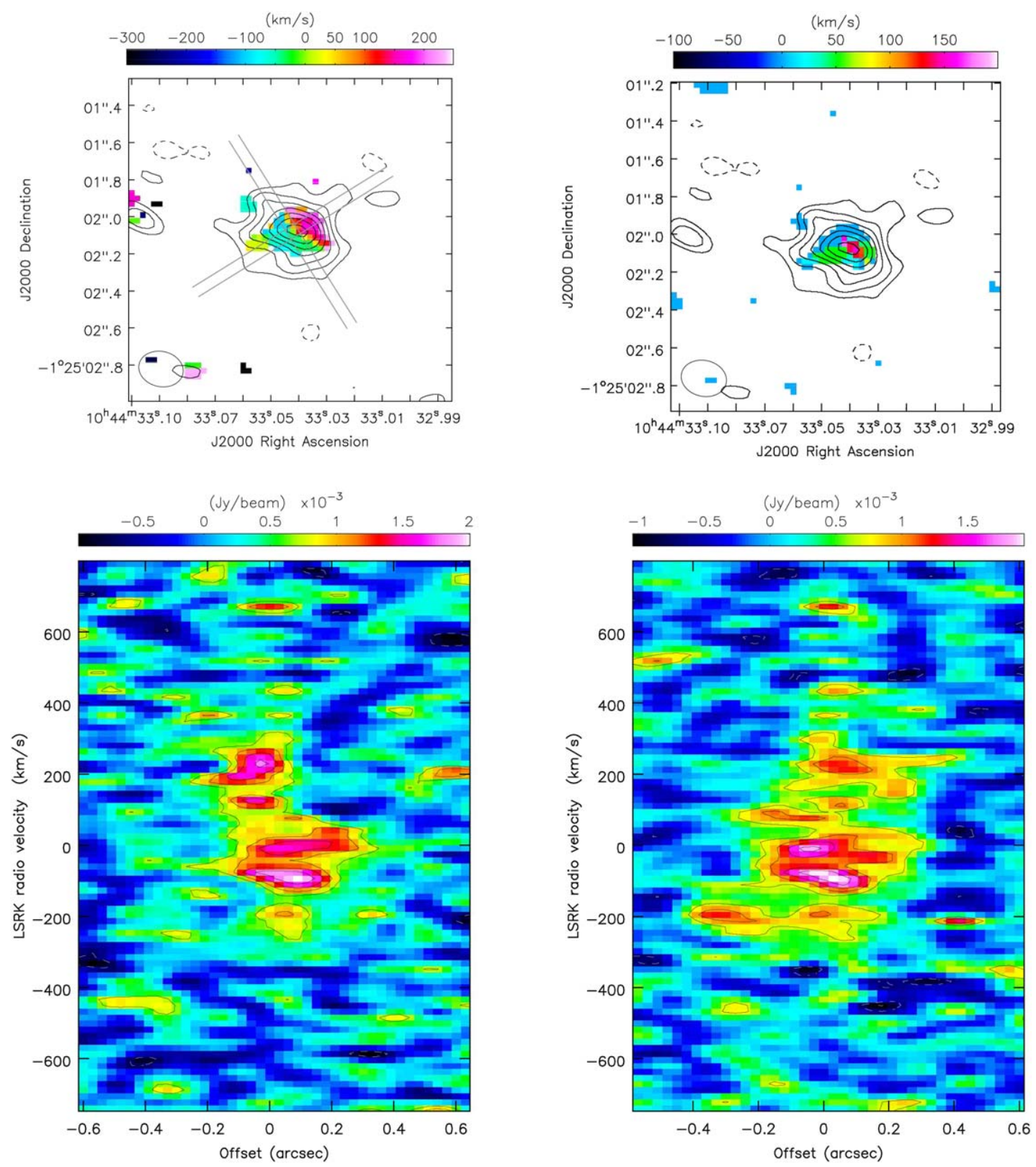

Figure 5. Upper panels show the intensity-weighted velocity (left) and velocity dispersion (right) maps of the [C II] line emission of SDSS J1044-0125, together with the line intensity map shown as contours (see Figure 4). The velocity and velocity dispersion maps are calculated using only the $>4 \sigma$ pixels of the channel image. The lower panels show the PV diagrams along the directions of $\mathrm{PA}=122^{\circ}$ (left) and PA $=32^{\circ}$ (right, marked as gray lines on the velocity map in the upper left panel). The contour levels are $[-2,2,3,4,5] \times 0.34 \mathrm{mJy}_{\text {beam }}^{-1}$. The zero velocity corresponds to the [C II] redshift of $z=5.7847$ (Wang et al. 2013 ). The central position for the PV diagrams is R.A. $10^{\mathrm{h}} 44^{\mathrm{m}} 33^{\mathrm{s}} .04$, decl. $-01^{\mathrm{d}} 25^{\prime} 02^{\prime \prime} 08$, and the slit width is $0 !^{\prime \prime} 09$.

\section{Analysis and Discussion}

\subsection{Surface Brightnesses and Ratios of the Resolved [C II] and Dust Emission}

The ALMA [C II] images of SDSS J0129-0035 and SDSS J1044-0125 probe the ISM in the nuclear regions on kiloparsec scales in these quasar-starburst systems. As we described in the previous section, the line and continuum emission are resolved from the two objects at 0!"2 (i.e., $\sim 1.2 \mathrm{kpc}$ ) resolution. The deconvolved FWHM source sizes of the [C II] line emission are $1.8 \pm 0.2$ and $2.6 \pm 0.5$ times larger than that of the continuum for SDSS J0129-0035 and SDSS J1044-0125, respectively (based on the results from imfit, Table 1). This is consistent with the results found in previous ALMA imaging of other starburst quasar host galaxies and SMGs (Wang et al. 2013; Díaz-Santos et al. 2016; Venemans et al. 2016, 2017c; Cooke et al. 2018; Gullberg et al. 2018; Rybak et al. 2019). In most of these systems, the [C II] emission is found to be 1.3 to $>2$ times more extended than that of the dust continuum emission (Cooke et al. 2018; Gullberg et al. 2018).

Based on the [C II] and dust continuum intensity maps, we compare the surface brightnesses as a function of radius in the nuclear region of the two systems (Figure 8). We also include ULAS J1319+0950 in the comparison, which is another FIR luminous quasar-starburst system and spatially resolved in [C II] and dust continuum emission at a similar resolution in our ALMA Cycle 1 program (Shao et al. 2017, see Section 1). Here we scale the continuum intensity map (Figure 1) with a factor 


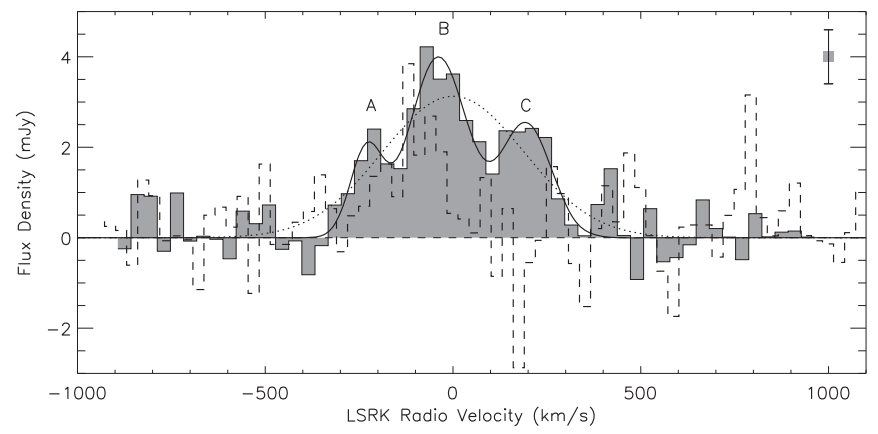

Figure 6. $[\mathrm{C} \mathrm{II}]$ line spectrum (gray histogram) integrated over the line-emitting region of SDSS J1044-0125 (i.e., within the $2 \sigma$ contour of the lower left panel of Figure 1). The error bar in the top right represents the $1 \sigma \mathrm{rms}$ for each channel. The dashed line is the spectrum of the CO (6-5) line emission (Wang et al. 2011), scaled by a factor of 2 . The zero velocity corresponds to the [C II] redshift of 5.7847 (Wang et al. 2013). The dotted line show a single-Gaussian fitting to the $[\mathrm{C} \mathrm{II}]$ line. The [C II] spectrum shows multiple peaks which cannot be well-fitted with a single-Gaussian component. Thus we also perform the fitting with three Gaussian components marked as A, B, and $\mathrm{C}$ in the spectrum and the solid line denotes the total emission of the three components (see Table 2).

Table 2

Spectral Fitting Parameters for SDSS J1044-0125

\begin{tabular}{lrcc}
\hline \hline Component & $\begin{array}{c}\text { Center } \\
\left(\mathrm{km} \mathrm{s}^{-1}\right)\end{array}$ & $\begin{array}{c}\text { FWHM } \\
\left(\mathrm{km} \mathrm{s}^{-1}\right)\end{array}$ & $\begin{array}{c}\text { Flux } \\
\left(\mathrm{Jy} \mathrm{km} \mathrm{s}^{-1}\right)\end{array}$ \\
\hline A & $-230 \pm 18$ & $103 \pm 40$ & $0.21 \pm 0.09$ \\
B & $-39 \pm 12$ & $180 \pm 43$ & $0.76 \pm 0.15$ \\
C & $194 \pm 19$ & $160 \pm 44$ & $0.42 \pm 0.11$ \\
\hline
\end{tabular}

of $L_{\mathrm{FIR}} / \mathrm{S}_{\mathrm{con}}$, where $L_{\mathrm{FIR}}$ is the FIR luminosity derived in Wang et al. (2013) assuming dust temperature of $47 \mathrm{~K}$ and emissivity index of $\beta=1.6$ (Beelen et al. 2006), and $S_{\text {con }}$ is the total continuum flux density close to the [C II] frequency in Table 1. This converts the monochromatic continuum map to a map of surface brightness of the FIR emission. We calculate the mean [C II] and FIR surface brightnesses within the rings at central radii of $0^{\prime \prime}, 0$ ! 1,0 ." 2,0 ." $3,0 . .4,0$. 5 , and $0 . " 6$ and a ring width of 0 ! 1 . The scale of 0 ! 1 corresponds to a physical size of $0.58 \mathrm{kpc}$ for ULAS J1319+0950, and $0.60 \mathrm{kpc}$ for SDSS J0129 -0035 and SDSS J1044-0125. The symbols and error bars shown in Figure 8 represent the mean and standard deviation of the pixel values in each ring. The [C II]-to-FIR surface brightness ratios are also plotted in the right panel of Figure 8. We model the surface brightness with an exponential disk in the two-dimensional image plane with a Sérsic index of $n=1$. We then convolve the model with the synthesized beam, calculate the mean surface brightnesses of the model in each ring described above, and fit these mean values to the data. The models are shown as dashed lines in Figure 8. They suggest that the [C II] and dust emission in the nuclear region of the quasar host galaxies follow an exponential light profile. The resolved surface brightness ratios between the [C II] and FIR dust emission range from 0.0001 to 0.001 within the central region where both $[\mathrm{C} \mathrm{II}]$ and dust continuum are detected.

In Figure 9, we compare the resolved [C II] and FIR continuum emission at different radii in these three $z \sim 6$ quasars with other systems on the [C II]-FIR ratio versus FIR surface brightness plot. We collect samples of star-forming galaxies and quasars at low and high redshifts that have continuum source size measurements from ALMA, including the GOALS sample of local luminous infrared galaxies (DíazSantos et al. 2013, 2017; Shangguan et al. 2019), high-redshift SMGs (Riechers et al. 2013; Neri et al. 2014; Gullberg et al. 2018), Lyman Break Galaxies (Capak et al. 2015; Jones et al. 2017; Hashimoto et al. 2019), and the $z \sim 6-7$ quasars (Wang et al. 2013; Díaz-Santos et al. 2016; Venemans et al. 2016, 2017c; Decarli et al. 2018). For the GOALS sample, we use the [C II]-FIR ratio extract from the central few kiloparsec region of the galaxies (i.e., the 9 ". $4 \times 9$ ". 4 region of one PACS detector element where the [C II] and continuum emission peaks, see details in Díaz-Santos et al. 2013, 2017). This is comparable to the [C II] and dust emission region detected in the high- $z$ samples. We recalculate the FIR luminosity surface brightness based on the GOALs sample catalog $^{21}$ and the decomposed infrared SED from Shangguan et al. (2019). For the high-redshift samples that have source size measurements from ALMA at $0 . " 6 \sim 0$.! 7 resolution, we adopt the FIR surface brightness averaged within the half-light radius of the continuum emitting region. We also include the resolved [C II] and FIR emission from two SMGs at $z=2.9$, which were imaged with ALMA at 0 ". 16 resolution and have FIR luminosities and source sizes comparable to the two quasar host galaxies we studied in this work (Rybak et al. 2019).

The continuum emission from SDSS J0129-0035 and SDSS J1044-0125, as well as ULAS J1319+0950, was resolved by ALMA on $1 \sim 2 \mathrm{kpc}$ scale and the peak FIR surface brightness is up to $\Sigma_{\text {FIR }} \sim 2 \times 10^{12} L_{\odot} \mathrm{kpc}^{-2}$. This is comparable to the highest values found in the compact SMGs and ULIRGs. As was shown in Figure 9, at this FIR surface brightness, the [C II]-FIR ratio is typically an order of magnitude lower than that of normal star-forming galaxies. The [C II]-FIR ratios in the three quasar hosts get higher toward larger radii, and the FIR surface brightnesses decrease to a few $10^{10} L_{\odot} \mathrm{kpc}^{-2}$. Thus the radial change of the [C II]FIR ratios found with the three objects can be understood as a trend of decreasing [C II]-FIR ratio with increasing FIR surface brightness which was found in samples of IR luminous starforming systems (e.g., $\Sigma_{\text {FIR }} \geqslant$ a few $10^{10} L_{\odot} \mathrm{kpc}^{-2}$, HerreraCamus et al. 2018). This trend has been termed "[C II]-FIR deficit" and was discussed in a number of papers (HaileyDunsheath et al. 2010; Ivison et al. 2010; Stacey et al. 2010; Farrah et al. 2013; Gullberg et al. 2015b, 2018; Díaz-Santos et al. 2016; Lutz et al. 2016; Smith et al. 2017; Cooke et al. 2018; Herrera-Camus et al. 2018).

In the compact dusty starburst systems, the high FIR surface brightness reveals high star formation rate surface density with high gas density and strong UV radiation field. As was mentioned in Section 1, in such an environment, the [C II] emission could be suppressed due to several possible mechanisms, including, e.g., saturation of the [C II] transition when the gas is heated with temperatures above $91 \mathrm{~K}$, (Muñoz \& Oh 2016; Rybak et al. 2019), increase of dust absorption of UV photons with high ionization parameter and a decrease of photoelectric heating efficiency (Herrera-Camus et al. 2018), or reduced amount of $\mathrm{C}^{+}$medium with more carbon in the form of $\mathrm{CO}$ at high cloud surface density (Narayanan \& Krumholz 2017). Though a model study of the origin of the [C II]-FIR deficit is beyond the goals of this work, we can conclude that the low [C II]-FIR ratio and high FIR surface brightness found in the central region of the FIR luminous quasar

\footnotetext{
21 http://goals.ipac.caltech.edu/
} 

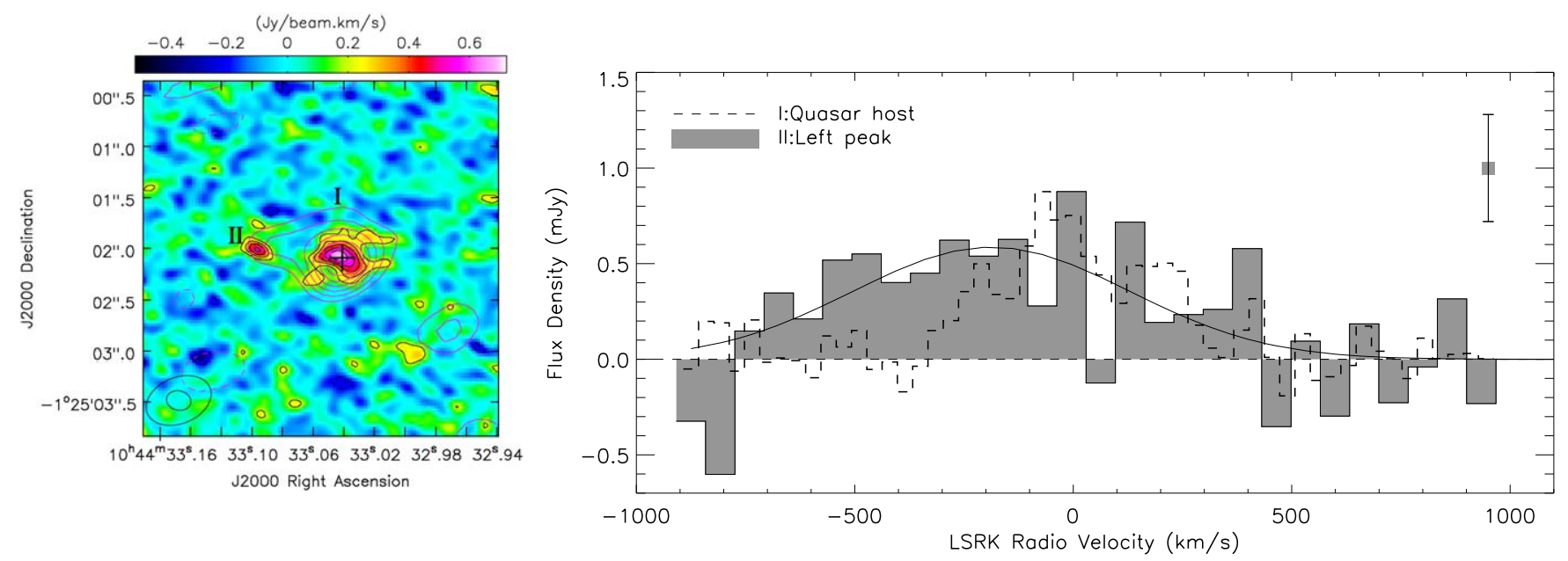

Figure 7. Left panel is a [C II] intensity map integrated over the velocity range of -740 to $400 \mathrm{~km} \mathrm{~s}^{-1}$, showing the component to the east of the quasar SDSS J1044 -0125. We mark the [C II] emission from the quasar host galaxy as component "I" and the east peak as component "II." The cross denotes the optical quasar position. The black contours are $[-3,-2,2,3,4,5,6] \times 0.1 \mathrm{Jy} \mathrm{beam}^{-1} \mathrm{~km} \mathrm{~s}^{-1}$. The red contours are the low-resolution ALMA Cycle 0 data of the [C II] velocity-integrated map published in Wang et al. (2013) with contour levels of $[-2,2,3,4,5,6,7,8] \times 0.14 \mathrm{Jy} \mathrm{beam}^{-1} \mathrm{~km} \mathrm{~s}^{-1}$. The low-resolution (FWHM beam size of 0 " $66 \times 0$ " 45 ) data show some extension to this east peak. The gray filled solid line in the right panel is the spectrum of the east peak, binned to a channel width of $67 \mathrm{~km} \mathrm{~s}^{-1}$. The typical $1 \sigma \mathrm{rms}$ noise of the spectrum is shown in the top right, and the solid line is the Gaussian line profile fit to the data. The dashed line shows the [C II] spectrum of the quasar (the same as Figure 6), scaled to the same peak level for comparison.
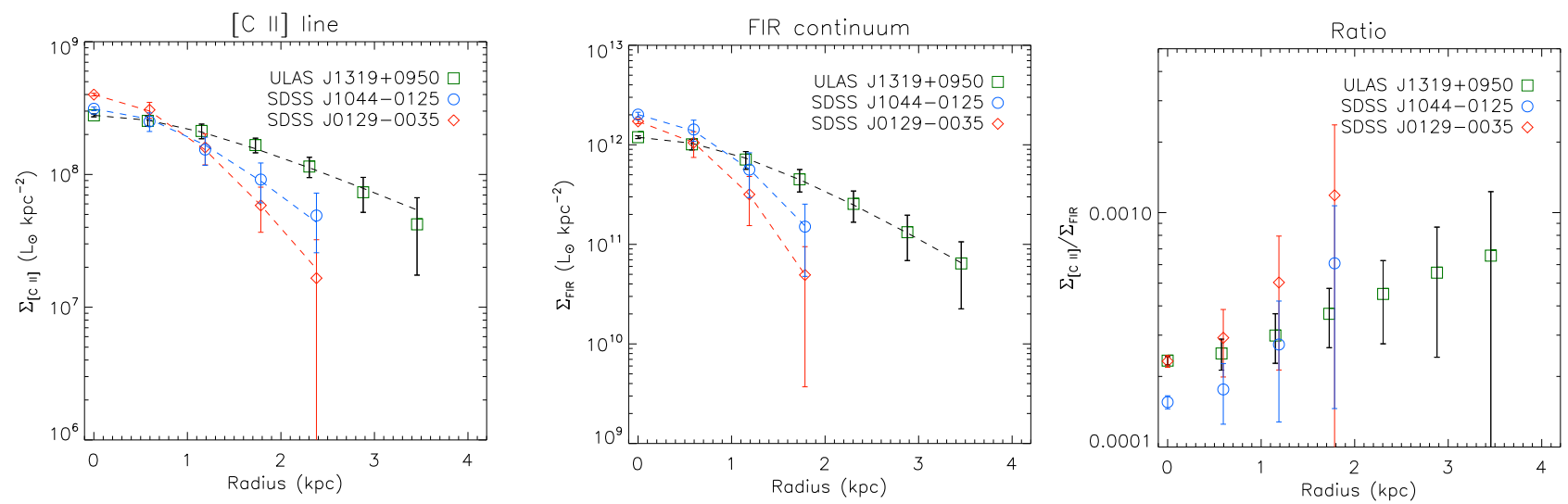

Figure 8. Surface brightnesses of the $[\mathrm{C} \mathrm{II}]$, dust continuum, and the $[\mathrm{C}$ II]-to-FIR surface brightness ratios as a function of distance from the center of three highredshift quasars, ULAS J1319+0950 (Shao et al. 2017), SDSS J0129-0035, and SDSS J1044-0125 (this work) that have been observed with ALMA at 0". $2 \sim 0$ 0! 3

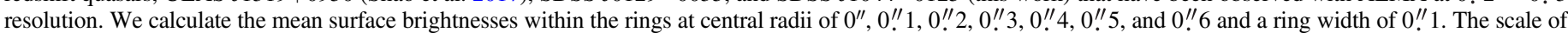
0 ." 1 corresponds to a physical size of $0.58 \mathrm{kpc}$ for ULAS J1319+0950, and $0.60 \mathrm{kpc}$ for SDSS J0129-0035 and SDSS J1044-0125. The error bars represent the standard deviation of the pixel values in each ring. The dashed lines represent an exponential disk model, convolved with the beam and fitted to the data.

hosts suggest a similar high density and dusty ISM heated by a high-intensity radiation field as was found in the compact and intense starburst systems.

Of the three objects, SDSS J1044-0125 and ULAS J1319 +0950 are about 10 times brighter than SDSS J0129-0035 in quasar UV luminosity (Jiang et al. 2009; Shen et al. 2019). However, their [C II]/FIR surface brightnesses and luminosity ratios are comparable. Though the central energetic active galactic nucleus (AGN) with a hard UV radiation field and strong X-ray emission may contribute to the ISM ionization and heating in the nuclear region (Stacey et al. 2010; Smith et al. 2017; Herrera-Camus et al. 2018; Shangguan et al. 2018), it is unlikely to be the dominant cause of the [C II]-FIR deficit in the quasar host galaxies (see also Decarli et al. 2018). As was pointed out by the spatially resolved studies of SMGs (Gullberg et al. 2018; Rybak et al. 2019) and local AGNs (Smith et al. 2017), the change of [C II]/FIR ratio with FIR surface brightness at different radii in the quasar host galaxies suggests that the $[\mathrm{CII}] /$ FIR deficit depends on the local conditions (radiation field, gas density, etc.), rather than the global properties of the systems.

We did not detect dust continuum emission at the [C II] peak to the east of the quasar SDSS J1044-0125. The continuum image sets a $3 \sigma$ upper limit of $0.12 \mathrm{mJy}$ for the flux density at the [C II] frequency. Assuming dust temperatures of 25 to $45 \mathrm{~K}$, this constrains the FIR luminosity of this component to be $<2 \times 10^{11} L_{\odot}$, and a [C II]-to-FIR luminosity ratio of $>2.4 \times 10^{-3}$ which is much higher than the central region of the quasar host. The high [C II]-to-FIR ratio of this component reveals ISM conditions different from that in the intense starburst nuclear region of the quasar host galaxy. Deep imaging of molecular $\mathrm{CO}$ and other fine structure line emission will help to measure the physical properties and excitation of the medium at this offset [C II] peak and address the nature of 


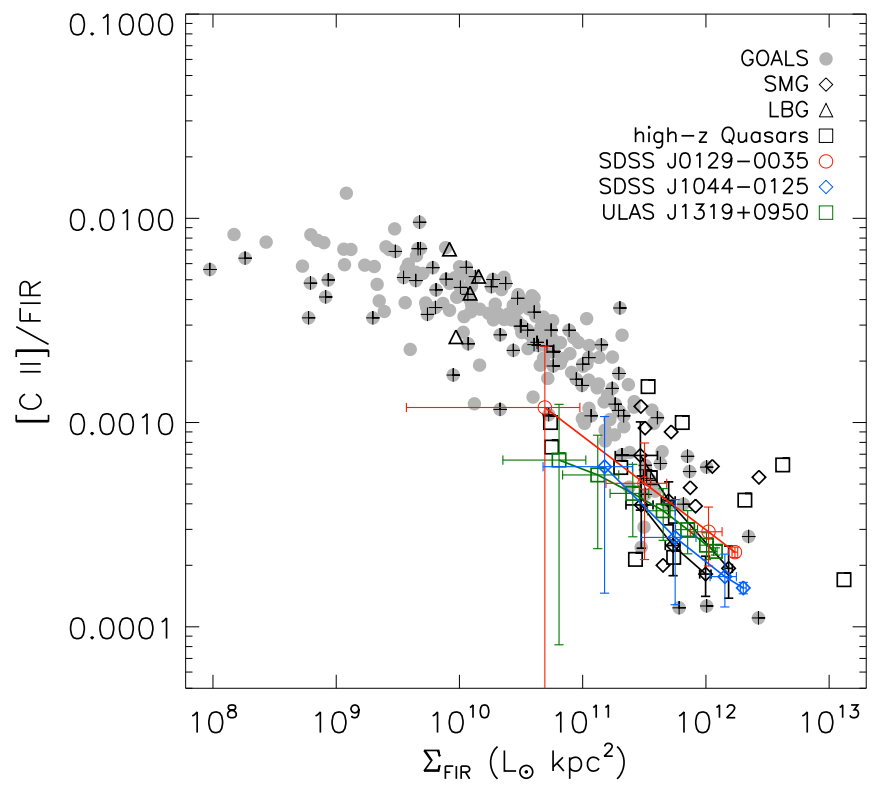

Figure 9. [C II]-FIR emission ratio vs. FIR surface brightness of the two $z=5.78$ quasars resolved by ALMA in this work. We also include ULAS J1319+0950 from Shao et al. (2017) which was resolved with ALMA at a similar resolution. We compare these FIR luminous $z \sim 6$ quasar hosts to other systems, including the GOALS sample of local luminous infrared galaxies (Díaz-Santos et al. 2013, 2017; Shangguan et al. 2019), the high-redshift submillimeter galaxies (SMGs; Riechers et al. 2013; Neri et al. 2014; Gullberg et al. 2018; Rybak et al. 2019), Lyman Break Galaxies (LBG; Capak et al. 2015; Jones et al. 2017; Hashimoto et al. 2019), and the $z \sim 6$ to 7 quasars (Wang et al. 2013; Díaz-Santos et al. 2016; Venemans et al. 2016, 2017c; Decarli et al. 2018). For the two $z=5.78$ quasars in this paper, ULAS $\mathrm{J} 1319+0950$, and the two compact SMG in Rybak et al. (2019) that have resolved measurements, we plot measurements at different radii (see Figure 8 above and Figure 8 in Rybak et al. 2019), connected by a line. For the GOALS sample, we use the [C II]/FIR ratio extract from the 9 !" $4 \times 9$ !" 4 region of one PACS detector element where the $[\mathrm{C}$ II] and continuum emission peaks, see details in Díaz-Santos et al. (2013, 2017). The plus sign indicates GOALS sources with possible AGN activity from emission line diagnostics, WISE colors, or SED decomposition (Shangguan et al. 2019).

this component (i.e., core of merging galaxy or node of outflow, etc., see more discussions below).

\subsection{Gas Kinematics in the Nuclear Region}

The [C II]-emitting gas in SDSS J0129-0035 shows clear velocity gradients, suggesting that most of the gas is likely from a rotating disk. The ordered motion constrains the dynamical mass of the quasar host galaxy within the [C II]emitting region (see details Section 4.3). The 0." 2 resolution [C II] image of SDSS J0129-0035 also reveals additional gas clumps, in particular, the components in the $\leqslant 0$ !! 2 region of the PV-diagram with velocities of $\sim \pm 200 \mathrm{~km} \mathrm{~s}^{-1}$ (Marked as Nos. 3 and 4 in the lower panel of Figure 3 ) and the two extended features as were described in Section 3. Though the individual tentative peaks with limited $(3 \sim 4 \sigma$, Figure 3$) \mathrm{S} / \mathrm{N}$ should be checked with deeper observations, we argue that the rotating velocity field with turbulent clumps reveals complex kinematics of the gas component in the nuclear region. Note that similar turbulent rotating gas disks were also reported with the $\mathrm{CO}(6-5)$ and [C II] images of SDSS J231038.88+185519.7 at $z=6.0$ (Feruglio et al. 2018) and the [C II] image of VIKING J030516.92-315056.0 at $z=6.6$ (Venemans et al. 2019) and was discussed in recent numerical simulations (e.g., Lupi et al. 2019); the gas around these powerful quasars shows significant dynamical evolution with substructures, including spirals, outflow and inflow clumps, and/or truncated disks.

The [C II] map of SDSS J1044-0125 does not show a clear sign of rotation. The PV diagrams (Figure 5) suggest a very turbulent gas velocity field in the central area; the gas within the 0 !" 2 region shows velocities ranging from -200 to $\gtrsim 200 \mathrm{~km} \mathrm{~s}^{-1}$. In addition, the [C II] line spectrum suggests multiple peaks (Table 2). Comparison between the $\mathrm{CO}(6-5)$ and $[\mathrm{C} \mathrm{II}] 158 \mu \mathrm{m}$ line spectra of this object (Figure 6) shows that the $\mathrm{CO}(6-5)$ line does not cover the same velocity range as that of the [C II] line. The high-J CO (e.g., CO (6-5), (7-6)) and [C II] lines in other millimeter-bright quasars at $z \sim 6$ usually show similar velocities and line widths (Riechers et al. 2009; Wang et al. 2013), suggesting that these lines trace similar kinematics of the ISM from the nuclear star-forming region. The case of SDSS J1044-0125 suggests a multiplephase ISM with different kinematic properties. The [C II] emission in galaxies is reported to trace the disk gas and starforming regions, as well as the warm diffuse neutral/ionized medium (Stacey et al. 2010; Pineda et al. 2014; Gullberg et al. 2015b; Croxall et al. 2017; Decarli et al. 2018; Lagache et al. 2018). [C II] emission in the quasar host galaxies could also be powered by the central powerful AGN (e.g., from the narrow line region, the X-ray dominated regions, etc.; Stacey et al. 2010; Uzgil et al. 2016). Asymmetric [C II] line emission that is broader than the molecular $\mathrm{CO}$ emission was detected by the radio galaxy $3 \mathrm{C} 326 \mathrm{~N}$, suggesting [C II] emission from a warm diffuse and turbulent molecular gas component powered by AGN-jet activity (Guillard et al. 2015a). On the other hand, the $\mathrm{CO}(6-5)$ line emission found in the high- $z$ quasar-starburst systems usually traces the dense molecular gas where stars are actively forming (Riechers et al. 2009; Greve et al. 2014; Liu et al. 2015; Venemans et al. 2017a). Thus, it is possible that, in SDSS J1044-0125, most of the [C II] emission covering a similar velocity range with the $\mathrm{CO}(6-5)$ line is from the intense star-forming regions, while the additional components in the velocity range without $\mathrm{CO}$ detection may be from more diffuse/warm medium. This mismatch between the $\mathrm{CO}$ and [C II] line spectra is a curious feature and should be checked with deeper and high-resolution imaging of the molecular line emission as well as other fine structure lines to address the complex physical condition, power resource, and kinematics of the ISM in this region.

The peak to the east of SDSS J1044-0125 reveals a [C II]emitting source with a very broad line width of FWHM = $750 \pm 190 \mathrm{~km} \mathrm{~s}^{-1}$ at a projected distance of $4.9 \mathrm{kpc}$ from the quasar (Component II in Figure 7). The nature of this offset component is unclear. Broad (line width up to $\geqslant 1000 \mathrm{~km} \mathrm{~s}^{-1}$ ), blue/redshifted, and low surface brightness [C II] emission was detected as gas outflows in the young quasar hosts at highredshift, which are evidence of strong AGN feedback (Cicone et al. 2014, 2015; Fiore et al. 2017; Bischetti et al. 2019). The offset peak we found in SDSS J1044-0125 could be a bright node of such an outflowing gas component. Companion [C II]/ submm continuum sources are detected close to other luminous quasars at high redshift (Decarli et al. 2017; Trakhtenbrot et al. 2017), revealing that these massive objects are in the centers of very active environments and that galaxy mergers may be a major triggering mechanism for SMBH and galaxy growth in these systems (Li et al. 2007; Narayanan et al. 2008, 2015; Volonteri et al. 2015; Watson et al. 2015; Valiante et al. 2017; Marrone et al. 2018). SDSS J1044-0125, as one of the most 

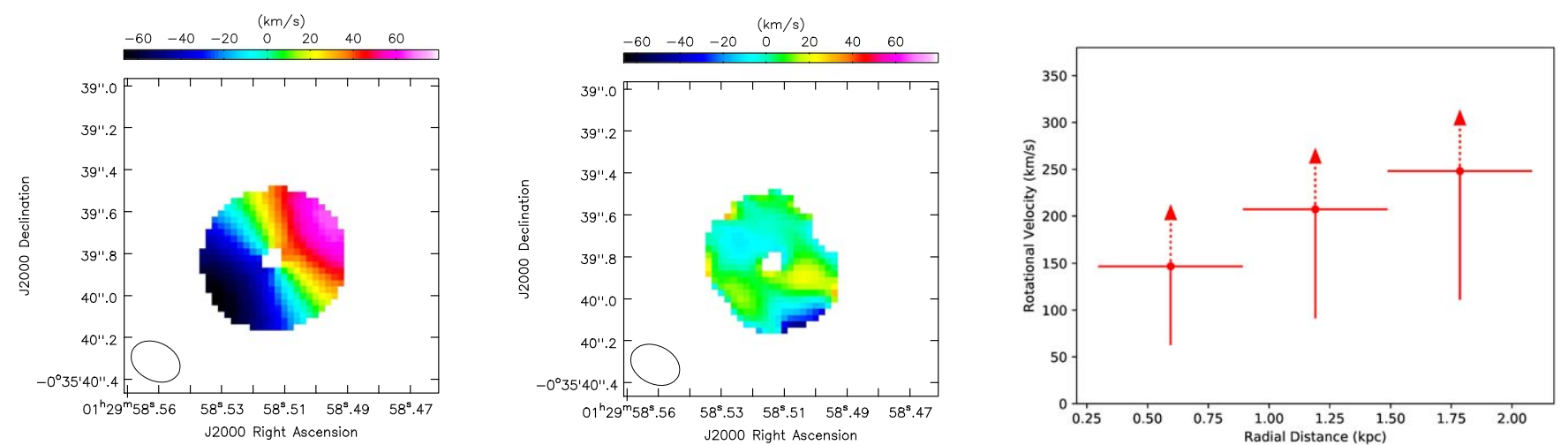

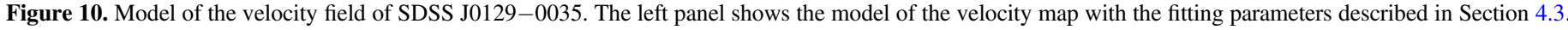

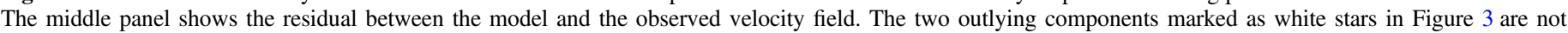

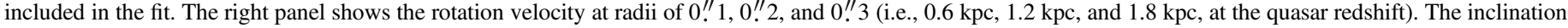

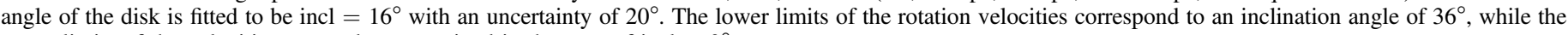
upper limits of the velocities cannot be constrained in the case of incl $=0^{\circ}$.

luminous quasars known at this redshift, may lie in a massive dark matter halo, and has already experienced several galaxy merger events (Li et al. 2007; Valiante et al. 2017; Marrone et al. 2018). Thus, it is also possible that this offset [C II] component is the dense core of a satellite galaxy that will merge with the quasar host.

In summary, the ALMA observations at 0.2 resolution reveal complex gas kinematics in the nuclear region of the quasar-starburst systems at $z \sim 6$, e.g., rotation and/or turbulent gas clumps. The turbulent gas kinematics, as well as the multiphase ISM suggested by the [C II] and CO emission in SDSS J1044-0125, indicate rich dynamical activities of ISM powered by the central AGN and/or the intense nuclear star formation. It will be interesting to expand the high resolution imaging studies to more examples to investigate the kinematic activities of the ISM in these extreme quasarstarburst systems. The nature of the peak to the east of SDSS J1044-0125 should also be checked with deeper observations of other emission lines to constrain the ISM properties and its dynamical connection to the quasar host.

\subsection{Constraints on the Host Galaxy Dynamics of SDSS J0129-0035}

We here model the gas velocity map of SDSS J0129-0035 with a simple circular rotating gas disk, following the method used in Shao et al. (2017; see also Jones et al. 2017), to constrain the gas dynamics in this system. We use the ROTCUR task in the Groningen Image Processing System (GIPSY3; van der Hulst et al. 1992) to fit the observed velocity field. As was described in Shao et al. (2017), the parameters for the model are (i) the sky coordinates of the rotational center of the disk, (ii) the system velocity $V_{\text {sys }}$, (iii) the circular velocity at the fitting radius $V_{\mathrm{c}}(R)$, (iv) the position angle of the major axis on the receding half of the disk, counterclockwise from the north direction, $\phi,(\mathrm{v})$ the inclination angle between the normal direction of the ring and line of sight, incl, and (vi) the azimuthal angle related to incl, $\phi$, and the disk center. We fix the disk center as the Gaussian peak center of the [C II] emission, and choose $V_{\text {sys }}=0 \mathrm{~km} \mathrm{~s}^{-1}$ to refer to the [C II] redshift of 5.7787 (Wang et al. 2013). We then use the data within a radius of 0 ". 35 to determine $\phi$ and incl. This is approximately the $2 \sigma$ contour region of the [C II] line emission (Figure 1). This fit gives $\phi$ and incl as $\phi=56 \pm 1^{\circ}$ and incl $=16 \pm 20^{\circ}$, respectively, where the error bars denote the fitting errors. This suggests a gas disk that is very close to faceon with incl $\leqslant 36^{\circ}$.

In Wang et al. (2013), we adopted the same inclined circular disk assumption and estimated the inclination angle from the deconvolved axis ratio of the Cycle 0 [C II] map, i.e., incl $=\arccos \left(a_{\text {minor }} / a_{\text {major }}\right)=56^{\circ}$. This is much larger than the value we obtained in this paper. It is likely that the two extended features (Nos. 1 and 2 in Figure 3) are unresolved in the low-resolution ALMA Cycle 0 image, which results in a larger major axis value, i.e., $a_{\text {major }}=0$ ". $41 \pm 0$ ". 06 from the Cycle 0 data compared to $a_{\text {major }}=0$ ". $32 \pm 0$ ". 03 in this work (Table 1). We cannot determine if these two features are part of the rotating disk or not; thus, we did not include those pixels in the fitting in this work.

Adopting the $\phi$ and incl values above, we constrain the circular velocities at radii of 0 !' 1,0 ." 2 , and $0 . " 3$ (Figure 10), corresponding to physical scales of $0.6,1.2$, and $1.8 \mathrm{kpc}$. If the inclination angle is fixed to $16^{\circ}$, the circular velocity at the largest radius (i.e., $1.8 \mathrm{kpc}$ ) is $V_{\mathrm{c}}=250 \mathrm{~km} \mathrm{~s}^{-1}$. Considering the fitting uncertainties of incl, we estimate a lower limit to the circular velocity of $V_{\mathrm{c}}=110 \mathrm{~km} \mathrm{~s}^{-1}$ with incl $=36^{\circ}$. An upper limit of the circular velocity cannot be constrained as incl could be very close to zero. Using $V_{\mathrm{c}}=250 \mathrm{~km} \mathrm{~s}^{-1}$, we derive the dynamical mass within a radius of $1.8 \mathrm{kpc}$ to be $M_{\text {dyn }}=2.6 \times 10^{10} M_{\odot}$. If the lower limit of the circular velocity of $110 \mathrm{~km} \mathrm{~s}^{-1}$ is adopted, the dynamical mass will decrease to $5.2 \times 10^{9} M_{\odot}$.

Based on the quasar $1450 \AA$ magnitude from the SDSS photometry (Jiang et al. 2009) and a $1450 \AA$ bolometric correction of $L_{\mathrm{bol}}=4.2 \times \lambda L_{\lambda 1450}$ (Runnoe et al. 2012), we derive a bolometric luminosity of $5.7 \times 10^{12} L_{\odot}$ for SDSS J0129-0035. Assuming that the SMBH is accreting at the Eddington limit, we estimate the $\mathrm{SMBH}$ mass of this object to be $M_{\mathrm{BH}}=1.7_{-0.9}^{+1.7} \times 10^{8} M_{\odot}$. We consider an uncertainty of 0.3 dex in the SMBH mass, which represents the scatter of the Eddington ratio of the $z \sim 6$ quasar sample (De Rosa et al. 2011). This results in an SMBH to host galaxy dynamical mass ratio of $M_{\mathrm{BH}} / M_{\mathrm{dyn}}=0.007$ with $V_{\mathrm{c}}=250 \mathrm{~km} \mathrm{~s}^{-1}$. If the lower limit of $V_{\mathrm{c}}=110 \mathrm{~km} \mathrm{~s}^{-1}$ is adopted, the mass ratio will become 0.03. Considering the facts that (i) the model has large uncertainties for face-on systems and cannot accurately determine the inclination angle and circular velocity, (ii) we do not reach the flat part of the rotation curve as shown in 


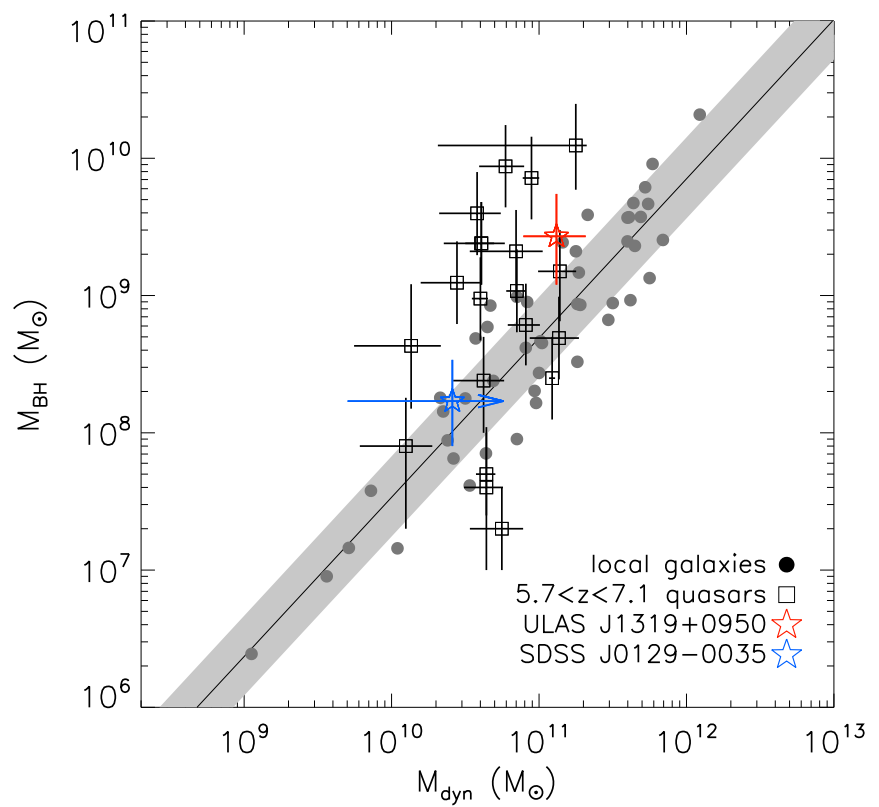

Figure 11. SMBH mass vs. host galaxy dynamical mass of the $z \sim 6$ to 7 quasars compared to the local galaxies. The black squares are the $z \sim 6$ to 7 quasars from the literature (Wang et al. 2013, 2016, 2019; Cicone et al. 2015; Willott et al. 2015, 2017; Venemans et al. 2016; Decarli et al. 2018; Feruglio et al. 2018; Izumi et al. 2018). These objects all have source size measurements from [C II] or $\mathrm{CO}$ observations and most of them have inclination angles estimated from the major and minor axis ratios (see details in Section 4.3). Two of these $z \sim 6$ quasars, SDSS J0129-0035 (this work) and ULAS J1319 +0950 (Shao et al. 2017) have [C II] emission resolved with ALMA at 0 ." $2 \sim 0$." 3 , showing clear velocity gradients. Disk dynamical modeling and fitting of inclination angle are performed based on the velocity maps. Based on this we obtained more reliable measurements of the host galaxy dynamical mass for ULAS J1319+0950 (Shao et al. 2017) and a lower limit for SDSS J0129-0035. We plot ULAS J1319+0950 and SDSS J0129-0035 as red and blue stars, respectively. The gray dots denote the sample of local galaxies and the solid line and the gray area represent the relationship of the local galaxies with \pm 0.3 dex intrinsic scatter (Kormendy \& Ho 2013).

Figure 10, and (iii) the outer part of the disk may not be traced by the [C II] velocity map due to low $\mathrm{S} / \mathrm{N}$ or because it is disrupted (Lupi et al. 2019; Venemans et al. 2019), the $M_{\text {dyn }}$ value derived above should be considered as a lower limit of the host galaxy dynamical mass for SDSS J0129-0035. The corresponding $M_{\mathrm{BH}} / M_{\mathrm{dyn}}$ ratio hence represents an upper limit.

We compare the SMBH mass and the lower limit of the host galaxy dynamical mass of SDSS J0129-0035 to those of other quasars at $z \sim 6-7$ quasars and the relationship of local galaxies (Kormendy \& Ho 2013). We collect the dynamical mass measurements of the $z \sim 6-7$ quasars from the literature. These objects are all detected in [C II] and/or CO line emission (Wang et al. 2013, 2016, 2019; Cicone et al. 2015; Willott et al. 2015, 2017; Venemans et al. 2016; Decarli et al. 2018; Feruglio et al. 2018; Izumi et al. 2018) and have source size measurements from [C II] or $\mathrm{CO}$ data and inclination angles estimated from the minor and major axis ratios of the line emission. The two objects, SDSS J0129-0035 (this work) and ULAS J1319+0950 (Shao et al. 2017), were observed with ALMA at a much better resolution of 0 ". $2 \sim 0$ !' 3 . The [C II] emission is resolved and the velocity maps show clear velocity gradients. These, combined with the disk dynamical model described above, provide more reliable constraints on the disk inclination angle, circular velocity, and dynamical mass of the quasar host galaxies. We denote these two objects as blue and red stars in the plot. According to Figure 11, the black hole-to-host mass relationship of local galaxies given by Kormendy \& Ho (2013) predicts a mass ratio of 0.0043 for an SMBH mass of $1.7 \times 10^{8} M_{\odot}$. Thus, it is unlikely that the black hole-to-host mass ratio of SDSS J0129-0035 is significantly above the local relationship. This is unlike the most luminous/massive quasars at this redshift, which usually show ratios a few to $\gtrsim 10$ times higher (Venemans et al. 2016; Decarli et al. 2018; Wang et al. 2019). This agrees with the results of other $[\mathrm{C} \mathrm{II}]$-detected $z \gtrsim 6$ quasars with similar or lower SMBH masses, in which the ratios are consistent with the trend defined by the local systems (Willott et al. 2010, 2017; Izumi et al. 2018). The dynamical mass constraints based on the $[\mathrm{C} \mathrm{II}]$ or $\mathrm{CO}$ imaging of these $z \sim 6-7$ quasars suggest that, in the early universe, the most massive SMBHs with masses of $10^{9}-10^{10} M_{\odot}$ may grow faster than that of their host galaxies (Walter et al. 2004; Wang et al. 2016, 2019; Venemans et al. 2016; Decarli et al. 2018) while the less massive systems $\left(10^{7} \sim 10^{8} M_{\odot}\right)$ are evolving more closely to the trend of local galaxies (Willott et al. 2017; Izumi et al. 2018).

\section{Summary}

Based on new ALMA observations we present images of the [C II] and dust continuum emission from the host galaxies of two $z=5.78$ quasars, SDSS J0129-0035 and SDSS J1044 -0125 on 0 ". 2 scales, resolving the ISM emission in the central $\sim 4 \mathrm{kpc}$ of the hosts. The main conclusions are summarized as follows:

1. The FWHM sizes of the [C II] emission is $1.8-2.4 \mathrm{kpc}$ and that of the dust continuum $\sim 1 \mathrm{kpc}$ for the two objects, constraining the size of the intense star-forming region around the accreting SMBHs. As was found in other starburst quasar host galaxies and SMGs at high redshift, the $[\mathrm{C} \mathrm{II}]$ emission regions appear to be more extended compared to that of dust continuum (Cooke et al. 2018; Gullberg et al. 2018).

2. We derive the [C II] and FIR surface brightness and emission ratios at different radii based on the resolved [C II] and continuum images. The resolved [C II]-FIR ratios are decreasing with increasing $\Sigma_{\mathrm{FIR}}$ toward the center, following the trend of the IR luminous starforming galaxies with $\Sigma_{\mathrm{FIR}}$ in the same range of a few $10^{10} L_{\odot} \mathrm{kpc}^{-2}$ to $2 \times 10^{12} L_{\odot} \mathrm{kpc}^{-2}$ (Stacey et al. 2010; Díaz-Santos et al. 2013; Gullberg et al. 2015b, 2018; Lutz et al. 2016; Cooke et al. 2018; Herrera-Camus et al. 2018; Rybak et al. 2019). The low [C II]-FIR ratios of $0.0001 \sim 0.0002$ found at the peak $\Sigma_{\text {FIR }}$ in the central region of these two quasar hosts suggest dense ISM with high-intensity radiation field similar to that in the compact dusty starburst systems (Díaz-Santos et al. 2017; Gullberg et al. 2018; Herrera-Camus et al. 2018; Rybak et al. 2019).

3. The velocity field and PV diagrams of SDSS J0129 -0035 reveal a combination of rotation with turbulent gas clumps; We detect clear velocity gradients in the velocity map. We also see features with velocity offsets of -190 and $+50 \mathrm{~km} \mathrm{~s}^{-1}$ at the outer part of the [C II] emitting region and gas clumps with velocity offsets of $\pm 200 \mathrm{~km} \mathrm{~s}^{-1}$ within the central 0 ". 2 region, suggesting other kinematic activities, such as inflow or outflows.

4. The $[\mathrm{CII}]$-emitting gas in the central region of SDSS J1044-0125 does not show a clear sign of rotation. The 
PV-diagram of the [C II] emission suggests very turbulent gas kinematics in the central area. The [C II] line spectrum shows multiple peaks, which is broader and slightly offset from the $\mathrm{CO}(6-5)$ line emission. In addition, we tentatively detect an offset peak, $4.9 \mathrm{kpc}$ away to the east of the quasar with a very broad line width $\left(750 \pm 190 \mathrm{~km} \mathrm{~s}^{-1}\right)$ which may relate to an infalling companion source or node of outflows.

5. Overall, the gas kinematics on kiloparsec scales in the nuclear region of the two starburst quasar host galaxies reveal significant dynamical evolution of the ISM, which could be powered by both AGN and the intense star formation.

6. Based on the dynamical modeling of the [C II] velocity gradients of SDSS J0129-0035, we derive a disk inclination angle very close to face-on (i.e., incl $=16^{\circ} \pm 20^{\circ}$ ). We can thus only put a lower limit on the host dynamical mass within the $[\mathrm{C} \mathrm{II}]$-emitting region. The result argues against an SMBH-bulge mass ratio that is significantly above (e.g., larger by a factor of 10) the value derived from the local relationship. As was discussed in previous studies of the less luminous/massive quasar population at $z \sim 6$, the mass ratio of SDSS J0129-0035 is likely to be closer to the local relationship than to that found for the most luminous and massive quasars at the same redshift (Willott et al. 2010; Venemans et al. 2016; Izumi et al. 2018; Wang et al. 2019).

The ALMA images of the [C II] emission in these young quasar host galaxies at kiloparsec resolution reveal rich features of gas kinematics in the nuclear region around the powerful AGNs. Between the two objects studied in this work, we do see more turbulent/clumpy gas in the nuclear region and a curious discrepancy between the [C II] and CO line profiles in SDSS J1044-0125, which hosts a more massive SMBH with higher quasar luminosity. However, it is still unclear if this is due to the feedback of the powerful AGN or nuclear star formation. If the east peak is associated with an infalling companion, the tidal perturbations could also affect the gas dynamic in the nuclear region. Deeper images of the [C II] and molecular $\mathrm{CO}$ emission from this object are still required to confirm these features. The high-resolution imaging studies should also be expanded to a larger sample to check for significant evolution of gas kinematic properties with AGN activity, e.g., more turbulent or disrupting gas disk in more luminous AGNs. Deep imaging of the molecular gas (e.g., with ALMA) and the stellar component (e.g., with the JWST in the future) on similar scales are also urgently required to fully probe host galaxy evolution of these young quasars at the earliest epochs.

This work was supported by National Key Program for Science and Technology Research and Development (grant 2016YFA0400703). We are thankful for the support from the National Science Foundation of China (NSFC) grant Nos. 11721303, 11373008, 11533001, 11473004, the Strategic Priority Research Program "The Emergence of Cosmological Structures" of the Chinese Academy of Sciences, grant No. XDB09000000, and the National Key Basic Research Program of China 2014CB845700. R.W. acknowledges support from the Thousand Youth Talents Program of China and the NSFC grant Nos. 11443002 and 11473004. D.R. acknowledges support from the National Science Foundation under grant No. AST-1614213. D.N. was funded in part by NSF AST-
1715206 and HST AR-15043.0001. The National Radio Astronomy Observatory (NRAO) is a facility of the National Science Foundation operated under cooperative agreement by Associated Universities, Inc. This paper makes use of the following ALMA data: ADS/JAO.ALMA\#2011.0.00206.S and \#2012.1.00240.S. ALMA is a partnership of ESO (representing its member states), NSF (USA) and NINS (Japan), together with NRC (Canada) and NSC and ASIAA (Taiwan), in cooperation with the Republic of Chile. The Joint ALMA Observatory is operated by ESO, AUI/NRAO, and NAOJ. R.W. thanks Dr Matus Rybak for providing the resolved [C II] and FIR continuum measurements of their SMG sample. R.W. thanks Dr Shangguan for providing FIR luminosities of the GOALS sample.

Facilities: ALMA.

\section{ORCID iDs}

Ran Wang (iD https://orcid.org/0000-0003-4956-5742 Yali Shao (iD https://orcid.org/0000-0002-1478-2598 Chris L. Carilli (i) https:// orcid.org/0000-0001-6647-3861 Gareth C. Jones (ib https://orcid.org/0000-0002-0267-9024 Fabian Walter (iD https://orcid.org/0000-0003-4793-7880 Xiaohui Fan (10 https://orcid.org/0000-0003-3310-0131 Dominik A. Riechers (iD https://orcid.org/0000-0001-9585-1462 Roberto Decarli (ib https://orcid.org/0000-0002-2662-8803 Frank Bertoldi (1D https://orcid.org/0000-0002-1707-1775 Michael A. Strauss (iD https://orcid.org/0000-0002-0106-7755 Alain Omont (iD https://orcid.org/0000-0002-4721-3922 Linhua Jiang (iD https://orcid.org/0000-0003-4176-6486 Desika Narayanan (iD https://orcid.org/0000-0002-7064-4309 Karl M. Menten (D) https://orcid.org/0000-0001-6459-0669 Bram P. Venemans (10 https://orcid.org/0000-0001-9024-8322

\section{References}

Bañados, E., Decarli, R., Walter, F., et al. 2015, ApJL, 805, 8 Bañados, E., Venemans, B. P., Decarli, R., et al. 2016, ApJS, 227, 11 Bañados, E., Venemans, B. P., Mazzucchelli, C., et al. 2018, Natur, 553, 473 Beelen, A., Cox, P., Benford, D. J., et al. 2006, ApJ, 642, 694 Bertoldi, F., Carilli, C. L., Cox, P., et al. 2003a, A\&A, 406, L55 Bertoldi, F., Cox, P., Neri, R., et al. 2003b, A\&A, 409, L47 Bischetti, M., Maiolino, R., Fiore, S., et al. 2019, A\&A, 630, A59 Capak, P. L., Carilli, C., Jones, G., et al. 2015, Natur, 522, 455 Carilli, C. L., Neri, R., Wang, R., et al. 2007, ApJL, 666, L9 Carilli, C. L., \& Walter, F. 2013, ARA\&A, 51, 105

Chehade, B., Carnall, A. C., Shanks, T., et al. 2018, MNRAS, 478, 1649 Cicone, C., Maiolino, R., Gallerani, S., et al. 2015, A\&A, 574, 14 Cicone, C., Maiolino, R., Sturm, E., et al. 2014, A\&A, 562, 21 Cooke, E. A., Smail, I., Swinbank, A. M., et al. 2018, ApJ, 861, 100 Croxall, K., Smith, J. D. T., Pellegrini, E., et al. 2017, ApJ, 845, 96 Decarli, R., Walter, F., Bañados, E., et al. 2017, Natur, 545, 457 Decarli, R., Walter, F., Venemans, B. P., et al. 2018, ApJ, 854, 97 De Rosa, G., Decarli, R., Walter, F., et al. 2011, ApJ, 739, 56 Díaz-Santos, T., Armus, L., Charmandaris, V., et al. 2013, ApJ, 774, 68 Díaz-Santos, T., Armus, L., Charmandaris, V., et al. 2017, ApJ, 846, 32 Díaz-Santos, T., Assef, R. J., Blain, A. W., et al. 2016, ApJL, 816, L6 Fan, X., Strauss, M. A., Richards, G. T., et al. 2006, AJ, 131, 1203 Fan, X., White, R. L., Davis, M., et al. 2000, AJ, 120, 1167 Farrah, D., Lebouteiller, V., Spoon, H. W. W., et al. 2013, ApJ, 776, 38 Feruglio, C., Fiore, F., Carniani, S., et al. 2018, A\&A, 619, A39 Fiore, F., Feruglio, C., Shankar, F., et al. 2017, A\&A, 601, 143 Graciá-Carpio, J., Sturm, E., Hailey-Dunsheath, S., et al. 2011, ApJL, 728, 7 Greve, T. R., Leonidaki, I., Xilouris, E. M., et al. 2014, ApJ, 794, 142 Guillard, P., Boulanger, F., Lehnert, M. D., et al. 2015a, A\&A, 574, 32 Gullberg, B., De Breuck, C., Vieira, J. D., et al. 2015b, MNRAS, 449, 2883 Gullberg, B., Swinbank, A. M., Smail, I., et al. 2018, ApJ, 859, 12 Hailey-Dunsheath, S., Nikola, T., Stacey, G. J., et al. 2010, ApJL, 714, L162 Hashimoto, T., Inoue, A. K., Mawatari, K., et al. 2019, PASJ, 71, 71 
Herrera-Camus, R., Bolatto, A. D., Wolfire, M. G., et al. 2015, ApJ, 800, 1 Herrera-Camus, R., Sturm, E., Graciá-Carpio, J., et al. 2018, ApJ, 861, 95 Ivison, R. J., Papadopoulos, P. P., Smail, Ian., et al. 2011, MNRAS, 412, 1913 Ivison, R. J., Swinbank, A. M., Swinyard, B., et al. 2010, A\&A, 518, 35 Izumi, T., Onoue, M., Shirakata, H., et al. 2018, PASJ, 70, 36 Jiang, L., Fan, X., Bian, F., et al. 2009, AJ, 138, 305

Jiang, L., McGreer, I. D., Fan, X., et al. 2016, ApJ, 833, 222

Jones, G. C., Carilli, C. L., Shao, Y., et al. 2017, ApJ, 850, 180

Kormendy, J., \& Ho, L. C. 2013, ARA\&A, 51, 511

Lagache, G., Cousin, M., \& Chatzikos, M. 2018, A\&A, 609, L130

Li, Y., Hernquist, L., Robertson, B., et al. 2007, ApJ, 665, 187

Liu, D., Gao, Y., Isaak, K., et al. 2015, ApJL, 810, L14

Luhman, M. L., Satyapal, S., Fischer, J., et al. 2003, ApJ, 594, 758

Lupi, A., Volonteri, M., Decarli, R., et al. 2019, MNRAS, 488, 4004

Lutz, D., Berta, S., Contursi, A., et al. 2016, A\&A, 591, A136

Maiolino, R., Cox, P., Caselli, P., et al. 2005, A\&A, 440, L51

Maiolino, R., Gallerani, S., Neri, R., et al. 2012, MNRAS, 425, L66

Malhotra, S., Kaufman, M. J., Hollenbach, D., et al. 2001, ApJ, 561, 766

Marrone, D. P., Spilker, J. S., Hayward, C. C., et al. 2018, Natur, 533, 51

Matsuoka, Y., Iwasawa, K., Onoue, M., et al. 2018a, ApJS, 237, 5

Matsuoka, Y., Onoue, M., Kashikawa, N., et al. 2016, ApJ, 828, 26

Matsuoka, Y., Onoue, M., Kashikawa, N., et al. 2018b, PASJ, 70, 35

Mortlock, D. J., Patel, M., Warren, S. J., et al. 2009, A\&A, 509, 97

Mortlock, D. J., Warren, S. J., Venemans, B. P., et al. 2011, Natur, 474, 616

Muñoz, J. A., \& Oh, S. P. 2016, MNRAS, 463, 2085

Narayanan, D., \& Krumholz, M. R. 2017, MNRAS, 467, 50

Narayanan, D., Li, Y., Cox, T. J., et al. 2008, ApJS, 174, 13

Narayanan, D., Turk, M., Feldmann, R., et al. 2015, Natur, 525, 496

Neri, R., Downes, D., Cox, P., \& Walter, F. 2014, A\&A, 562, 35

Omont, A., Willott, C. J., Beelen, A., et al. 2013, A\&A, 552, A43

Petric, A. O., Carilli, C. L., Bertoldi, F., et al. 2003, AJ, 126, 15

Pineda, J. L., Langer, W. D., \& Goldsmith, P. F. 2014, A\&A, 570, A121

Priddey, R. S., Isaak, K. G., McMahon, R. G., Robson, E. I., \& Pearson, C. P. 2003, MNRAS, 344, L74

Riechers, D. A., Bradford, C. M., Clements, D. L., et al. 2013, Natur, 496, 329

Riechers, D. A., Walter, F., Bertoldi, F., et al. 2009, ApJ, 703, 1338

Robson, I., Priddey, R. S., Isaak, K. G., \& McMahon, R. G. 2004, MNRAS, 351, L29
Runnoe, J. C., Brotherton, M. S., \& Shang, Z. 2012, MNRAS, 422, 478

Rybak, M., Calistro Rivera, G., Hodge, J. A., et al. 2019, ApJ, 876, 112

Shangguan, J., Ho, L. C., Li, R., et al. 2019, ApJ, 870, 104

Shangguan, J., Ho, L. C., \& Xie, Y. 2018, ApJ, 854, 158

Shao, Y., Wang, R., Jones, G. C., et al. 2017, ApJ, 845, 138

Shen, Y., Wu, J., Jiang, L., et al. 2019, ApJ, 873, 35

Smith, J. D. T., Croxall, K., Draine, B., et al. 2017, ApJ, 834, 5

Solomon, P. M., \& Vanden Bout, P. A. 2005, ARA\&A, 43, 677

Spergel, D. N., Bean, R., Doré, O., et al. 2007, ApJS, 170, 377

Stacey, G. J., Hailey-Dunsheath, S., Ferkinhoff, C., et al. 2010, ApJ, 724, 957

Trakhtenbrot, B., Lira, P., Netzer, H., et al. 2017, ApJ, 836, 8

Uzgil, B. D., Bradford, C. M., Hailey-Dunsheath, S., Maloney, P. R., \& Aguirre, J. E. 2016, ApJ, 832, 209

Valiante, R., Agarwal, B., Habouzit, M., \& Pezzulli, E. 2017, PASA, 34, 31

van der Hulst, J. M., Terlouw, J. P., Begeman, K. G., Zwitser, W., \& Roelfsema, P. R. 1992, ASPC, 25, 131

Venemans, B. P., Findlay, J. R., Sutherland, W. J., et al. 2013, ApJ, 779, 24

Venemans, B. P., Neeleman, M., Walter, F., et al. 2019, ApJL, 874, 30

Venemans, B. P., Walter, F., Decarli, R., et al. 2017a, ApJ, 845, 154

Venemans, B. P., Walter, F., Decarli, R., et al. 2017b, ApJL, 851, 8

Venemans, B. P., Walter, F., Decarli, R., et al. 2017c, ApJ, 837, 146

Venemans, B. P., Walter, F., Zschaechner, L., et al. 2016, ApJ, 816, 37

Volonteri, M., Silk, J., \& Dubus, G. 2015, ApJ, 804, 148

Walter, F., Carilli, C. L., Bertoldi, F., et al. 2004, ApJL, 615, L17

Walter, F., Riechers, D., Cox, P., et al. 2009, Natur, 457, 699

Wang, F., Wang, R., Fan, X., et al. 2019, ApJ, 880, 2

Wang, R., Carilli, C., Beelen, A., et al. 2007, AJ, 134, 617

Wang, R., Carilli, C., Neri, R., et al. 2010, ApJ, 714, 699

Wang, R., Carilli, C., Wagg, J., et al. 2008, ApJ, 687, 848

Wang, R., Wagg, J., Carilli, C., et al. 2011, AJ, 142, 101

Wang, R., Wagg, J., Carilli, C., et al. 2013, ApJ, 773, 44

Wang, R., Wu, X.-B., Neri, R., et al. 2016, ApJ, 830, 53

Watson, D. F., Hearin, A. P., Berlind, A. A., et al. 2015, MNRAS, 446, 651

Willott, C. J., Albert, L., Arzoumanian, D., et al. 2010, AJ, 140, 546

Willott, C. J., Bergeron, J., \& Omont, A. 2015, ApJ, 801, 123

Willott, C. J., Bergeron, J., \& Omont, A. 2017, ApJ, 850, 108

Willott, C. J., Omont, A., \& Bergeron, J. 2013, ApJ, 770, 13

Wu, X.-B., Wang, F., Fan, X., et al. 2015, Natur, 518, 512 